\title{
Exponential decay of the viscoelastic wave equation of Kirchhoff type with a nonlocal dissipation
}

\author{
Mohamed Mellah and Ali Hakem
}

Abstract. The following viscoelastic wave equation of Kirchhoff type with nonlinear and nonlocal damping

$$
u_{t t}-\psi\left(\|\nabla u\|_{2}^{2}\right) \Delta u-\alpha \Delta u_{t}+\int_{0}^{t} g(t-\tau) \Delta u(\tau) d \tau+M\left(\|\nabla u\|_{2}^{2}\right) u_{t}=f(u),
$$

where $M(r)$ is a $C^{1}([0, \infty))$-function satisfying $M(r) \geq m_{1}>0$ for $r \geq 0$, is considered in a bounded domain $\Omega$ of $\mathbb{R}^{N}$. The existence of global solutions and decay rates of the energy are proved.

Mathematics Subject Classification (2010): 35L05, 35L70, 93D15.

Keywords: Kirchhoff equation, nonlocal damping, global solution, energy decay, relaxation function.

\section{Introduction}

In this paper, we shall consider the initial boundary value problem for the following integro-differential problem

$$
\left\{\begin{array}{l}
u_{t t}-\psi\left(\|\nabla u\|_{2}^{2}\right) \Delta u-\alpha \Delta u_{t}+\int_{0}^{t} g(t-\tau) \Delta u(\tau) d \tau \\
+M\left(\|\nabla u\|_{2}^{2}\right) u_{t}=f(u), \quad \text { in } \quad \Omega \times(0, T), \\
u(x, 0)=u_{0}(x), \quad u_{t}(x, 0)=u_{1}(x), \quad x \in \Omega, \\
u(x, t)=0, \quad x \in \partial \Omega, t>0,
\end{array}\right.
$$

where $\Omega$ is a bounded domain in $\mathbb{R}^{N}(N \geq 1)$ with smooth boundary $\partial \Omega$ so that the divergence theorem can be applied. $\psi(r)$ is a positive locally Lipschitz function satisfying $\psi(r) \geq m_{0}>0$, for $r \geq 0$ like $\psi(r)=m_{0}+b r^{\gamma}, b \geq 0$ and $\gamma \geq 1 . M(r)$ is a $C^{1}[0, \infty)$-function satisfying $M(r) \geq m_{1}>0$ for $r \geq 0$, the scalar function $g(s)$ (so-called relaxation kernel) is assumed to satisfy (2.1) and $f$ is a non-linear function 
as similar to $|u|^{p-2} u, p>2$. Here $\alpha \geq 0$. The motivation for this problem comes from the following original equation

$$
\rho h \frac{\partial^{2} u}{\partial t^{2}}+\delta \frac{\partial u}{\partial t}=\left\{p_{0}+\frac{E h}{L} \int_{0}^{L}\left(\frac{\partial u}{\partial x}\right)^{2} d x\right\} \frac{\partial^{2} u}{\partial x^{2}}+f,
$$

where $0 \leq x \leq L, t \geq 0$ and $u=u(x, t)$ is the lateral displacement at the space coordinate $x$ and the time $t, \rho$ the mass density, $h$ the cross-section area, $L$ the length, $E$ the Youngs modulus, $p_{0}$ the initial axial tension, $\delta$ the resistance modulus and $f$ the external force. When $\delta=f=0$, the equation (1.2) was first introduced by Kirchhoff [2].

In the absence of the term $M\left(\|\nabla u\|_{2}^{2}\right) u_{t}$. Wu and Tsai [7] studied (1.1) with $\alpha=1$. The authors established the global existence and energy decay under the assumption $g^{\prime}(t) \leq-r g(t), \forall t \geq 0$ for some $r>0$. Recently, this decay estimate of the energy function was improved by $\mathrm{Wu}$ in [6] under a weaker condition on $g$ i.e. $g^{\prime}(t) \leq 0$, $\forall t \geq 0$.

If we consider (1.1) with $[\psi \equiv 1, f=\alpha=0]$ and the bi-harmonic instead of Laplace operator one we get the model

$$
u_{t t}+\Delta^{2} u-\int_{0}^{t} g(t-\tau) \Delta^{2} u(\tau) d \tau+M\left(\|\nabla u\|_{2}^{2}\right) u_{t}=0 .
$$

Cavalcanti et al. [1] investigated the global existence, uniqueness and stabilization of energy. By taking a bounded or unbounded open set $\Omega$, the authors showed that the energy goes to zero exponentially provided that g goes to zero at the same form.

The main interest of the present paper is to examine whether there exists a global solution $u$ to (1.3) under the presence of the nonlinear and nonlocal dissipation represented by $M\left(\int_{\Omega}|\nabla u(x, t)|^{2} d x\right) u_{t}$ and the real-value function $M:[0,+\infty) \rightarrow\left[m_{1},+\infty\right)$, where $m_{1}>0$ will be considered of class $C^{1}$.

This kind of damping effect was firstly introduced by H. Lange and G. Perla Menzala [3] for the beam equation where the following model was considered

$$
u_{t t}+\Delta^{2} u+M\left(\int_{\Omega}|\nabla u(x, t)|^{2} d x\right) u_{t}=0 \quad \text { in } \quad \mathbb{R}^{N} \times \mathbb{R}^{+} .
$$

The nonlocal nonlinearity $M\left(\int_{\Omega}|\nabla u(x, t)|^{2} d x\right) u_{t}$ is indeed a damping term. It models a frictional mechanism acting on the body that depends on the average of $u$ itself. Moreover, if such $u$ does exist, we intend to investigate its asymptotic behavior as $t \rightarrow \infty$.

In this paper we show that under some conditions the solution is global in time and the energy decays exponentially. We first use Faedo-Galerkin method to study the existence of the simpler problem (3.1). Then, we obtain the local existence Theorem 3.2 by using contraction mapping principle. We obtain global existence of the solutions of (1.1) given in Theorem 4.4. Our technique of proof is similar to the one in [7] with some necessary modifications due to the nature of the problem treated here. Moreover, the asymptotic behavior of global solutions is investigated under some assumptions on the initial data. 


\section{Preliminaries}

In this section we present some assumptions, notations and Lemmas. We first make the following hypotheses.

(A1) $g: \mathbb{R}^{+} \rightarrow \mathbb{R}^{+}$is a bounded $C^{1}$ function satisfying

$$
\begin{gathered}
\int_{0}^{\infty} g(\tau) d \tau=l_{1}>0, \quad g(0)-K_{1} \int_{0}^{\infty} g(\tau) d \tau=l_{2}>0 \\
-K_{1} g(t) \leq g^{\prime}(t) \leq-K_{2} g(t)
\end{gathered}
$$

here $K_{1}$ and $K_{2}$ are positive constants.

(A2) $f(0)=0$ and there is a positive constant $K_{3}$ such that

$$
|f(u)-f(v)| \leq K_{3}|u-v|\left(|u|^{p-2}+|v|^{p-2}\right) \quad \text { for } \quad u, v \in \mathbb{R},
$$

and

$$
2<p<\infty \quad \text { if } \quad N=1,2 \quad \text { and } \quad 2<p \leq \frac{2(N-1)}{N-2} \quad \text { if } \quad N \geq 3 .
$$

(A3) The function $M(r)$ for $r \geq 0$ belongs to the class $C^{1}[0, \infty)$ and satisfies

$$
M(r) \geq m_{1}>0 \quad \text { for } \quad r \geq 0 .
$$

For functions $u(x, t), v(x, t)$ defined on $\Omega$, we introduce

$$
\begin{gathered}
(u, v)=\int_{\Omega} u v d x, \quad\|u\|_{2}=\left(\int_{\Omega}|u|^{2} d x\right)^{\frac{1}{2}}, \quad\|u\|_{\infty}=e s s \sup _{x \in \Omega}|u(x)|, \\
\|u\|_{p}=\left(\int_{\Omega}|u|^{p} d x\right)^{\frac{1}{p}}, \quad\|u\|_{H^{m}}=\left(\sum_{|\beta| \leq m}\left\|D^{\beta}\right\|_{2}^{2}\right)^{\frac{1}{2}} .
\end{gathered}
$$

Lemma 2.1. (Sobolev-Poincaré inequality [5]) If $2 \leq p \leq \frac{2 N}{N-2}$, then

$$
\|u\|_{p} \leq B_{1}\|\nabla u\|_{2}
$$

for $u \in H_{0}^{1}(\Omega)$ holds with some constant $B_{1}$.

\section{Local existence of solution}

In this section, we shall discuss the local existence of solutions for (1.1) by using contraction mapping principle. An important step in the proof of local existence Theorem 3.2 below is the study of the following simpler problem:

$$
\left\{\begin{array}{l}
u_{t t}-\mu(t) \Delta u-\alpha \Delta u_{t}+\int_{0}^{t} g(t-\tau) \Delta u(\tau) d \tau \\
\quad+\chi(t) u_{t}=f_{1}(x, t), \quad \text { in } \Omega \times(0, T), \\
u(x, 0)=u_{0}(x), \quad u_{t}(x, 0)=u_{1}(x), \quad x \in \Omega \\
u(x, t)=0, \quad x \in \partial \Omega, t \geq 0 .
\end{array}\right.
$$


Here, $T>0, \alpha \geq 1, f_{1}$ is a fixed forcing term in $\Omega \times(0, T), \mu(t)$ is a positive locally Lipschitz function on $[0, \infty)$ with $\mu(t) \geq m_{0}>0$ for $t \geq 0$ and $\chi(t)$ is $C^{1}$-function on $[0, \infty)$ such that $\chi(t) \geq 0$ for $t \geq 0$.

Lemma 3.1. Suppose that $(A 1)$ holds, and that $u_{0} \in H^{2}(\Omega) \cap H_{0}^{1}(\Omega), u_{1} \in H_{0}^{1}(\Omega)$ and $f_{1} \in L^{2}\left([0, T] ; L^{2}(\Omega)\right)$ be given. Then the problem (3.1) admits a unique solution $u$ such that

$$
\begin{gathered}
u \in C\left([0, T] ; H^{2}(\Omega) \cap H_{0}^{1}(\Omega)\right), \quad u_{t} \in C\left([0, T] ; L^{2}(\Omega)\right) \cap L^{2}\left([0, T] ; H_{0}^{1}(\Omega)\right), \\
u_{t t} \in L^{2}\left([0, T] ; L^{2}(\Omega)\right) .
\end{gathered}
$$

Proof. Let $\left(\omega_{n}\right)_{n \in \mathbb{N}}$ be a basis in $H^{2}(\Omega) \cap H_{0}^{1}(\Omega)$ and $V^{n}$ be the space generated by $\omega_{1}, \ldots, \omega_{n}, n=1,2, \cdots$. Let us consider

$$
u^{n}(t)=\sum_{k=1}^{n} d_{k}^{n}(t) w_{k},
$$

be the weak solution of the following approximate problem corresponding to (3.1)

$$
\begin{aligned}
& \int_{\Omega} u_{t t}^{n}(t) \omega d x+\mu(t) \int_{\Omega} \nabla u^{n}(t) \cdot \nabla \omega d x \\
& -\int_{0}^{t} g(t-\tau) \int_{\Omega} \nabla u^{n}(\tau) \cdot \nabla \omega d x d \tau+\alpha \int_{\Omega} \nabla u_{t}^{n}(t) \cdot \nabla \omega d x \\
& +\chi(t) \int_{\Omega} u_{t}^{n}(t) \omega d x=\int_{\Omega} f_{1}(x, t) w d x \quad \text { for } \omega \in V^{n},
\end{aligned}
$$

with initial conditions

$$
\begin{gathered}
u^{n}(0)=u_{0}^{n}=\sum_{k=1}^{n} \int_{\Omega} u_{0} w_{k} d x w_{k} \longrightarrow u_{0} \text { in } H^{2}(\Omega) \cap H_{0}^{1}(\Omega), \\
u_{t}^{n}(0)=u_{1}^{n}=\sum_{k=1}^{n} \int_{\Omega} u_{1} w_{k} d x w_{k} \longrightarrow u_{1} \text { in } H_{0}^{1}(\Omega) .
\end{gathered}
$$

By standard methods in differential equations, we prove the existence of solutions to $(3.2)-(3.4)$ on some interval $\left[0, t_{n}\right), 0<t_{n}<T$. In order to extend the solution of $(3.2)-(3.4)$ to the whole interval $[0, T]$, we need the following a priori estimate.

Step 1. (The first priori estimate) Replacing $w$ by $2 u_{t}^{n}(t)$ in (3.2), we have

$$
\begin{aligned}
& \frac{d}{d t}\left[\left\|u_{t}^{n}(t)\right\|_{2}^{2}+\mu(t)\left\|\nabla u^{n}(t)\right\|_{2}^{2}\right]+2 \alpha\left\|\nabla u_{t}^{n}(t)\right\|_{2}^{2}+2 \chi(t)\left\|u_{t}^{n}(t)\right\|_{2}^{2} \\
& \quad=\mu^{\prime}(t)\left\|\nabla u^{n}(t)\right\|_{2}^{2}+2 \int_{0}^{t} g(t-\tau) \int_{\Omega} \nabla u^{n}(\tau) \cdot \nabla u_{t}^{n}(t) d x d \tau \\
& \quad+2 \int_{\Omega} f_{1}(x, t) u_{t}^{n}(t) d x \leq \mu^{\prime}(t)\left\|\nabla u^{n}(t)\right\|_{2}^{2}+\left\|\nabla u_{t}^{n}(t)\right\|_{2}^{2} \\
& \quad+\|g\|_{L^{1}} \int_{0}^{t} g(t-\tau)\left\|\nabla u^{n}(\tau)\right\|_{2}^{2} d \tau+\left\|f_{1}\right\|_{2}^{2}+\left\|u_{t}^{n}(t)\right\|_{2}^{2} .
\end{aligned}
$$


Then, integrating (3.5) from 0 to $t$, we get

$$
\begin{aligned}
& \left\|u_{t}^{n}(t)\right\|_{2}^{2}+\mu(t)\left\|\nabla u^{n}(t)\right\|_{2}^{2}+(2 \alpha-1) \int_{0}^{t}\left\|\nabla u_{\tau}^{n}(\tau)\right\|_{2}^{2} d \tau \leq c_{1} \\
& +\int_{0}^{t}\left[1+\frac{1}{\mu(\tau)}\left(\left|\mu^{\prime}(\tau)\right|+\|g\|_{L^{1}}^{2}\right)\right]\left[\left\|u_{\tau}^{n}(\tau)\right\|_{2}^{2}+\mu(\tau)\left\|\nabla u^{n}(\tau)\right\|_{2}^{2}\right] d \tau,
\end{aligned}
$$

where

$$
c_{1}=\left\|u_{1}^{n}\right\|_{2}^{2}+\mu(0)\left\|\nabla u_{0}^{n}\right\|_{2}^{2}+\int_{0}^{t}\left\|f_{1}\right\|_{2}^{2} d t .
$$

Taking into account (3.3) and (3.4), we obtain from Gronwall's Lemma the first priori estimate

$$
\left\|u_{t}^{n}(t)\right\|_{2}^{2}+\mu(t)\left\|\nabla u^{n}(t)\right\|_{2}^{2}+\int_{0}^{t}\left\|\nabla u_{t}^{n}(\tau)\right\|_{2}^{2} d \tau \leq L_{1},
$$

for all $t \in[0, T]$. Here $L_{1}$ is a positive constant independent of $n \in \mathbb{N}$ and $t \in[0, T]$.

Step 2. (The second priori estimate) Replacing $\omega$ by $u_{t t}^{n}(t)$ in (3.2), we have

$$
\begin{aligned}
& \frac{d}{d t}\left[\mu(t) \int_{\Omega} \nabla u^{n}(t) \cdot \nabla u_{t}^{n}(t) d x+\frac{\alpha}{2}\left\|\nabla u_{t}^{n}(t)\right\|_{2}^{2}+\frac{\chi(t)}{2}\left\|u_{t}^{n}(t)\right\|_{2}^{2}\right] \\
& \quad+\left\|u_{t t}^{n}(t)\right\|_{2}^{2}=\mu^{\prime}(t) \int_{\Omega} \nabla u^{n}(t) \cdot \nabla u_{t}^{n}(t) d x+\mu(t)\left\|\nabla u_{t}^{n}(t)\right\|_{2}^{2} \\
& \quad+\frac{\chi^{\prime}(t)}{2}\left\|u_{t}^{n}(t)\right\|_{2}^{2}+\frac{d}{d t}\left(\int_{0}^{t} g(t-\tau) \int_{\Omega} \nabla u^{n}(\tau) \cdot \nabla u_{t}^{n}(t) d x d \tau\right) \\
& \left.\quad-g(0) \int_{\Omega} \nabla u^{n}(t) \cdot \nabla u_{t}^{n}(t) d x+\int_{\Omega} f_{1}(x, t) u_{t t}^{n}(t)\right) d x \\
& \quad-\int_{0}^{t} g^{\prime}(t-\tau) \int_{\Omega} \nabla u^{n}(\tau) \cdot \nabla u_{t}^{n}(t) d x d \tau .
\end{aligned}
$$

By $(A 1)$, Hölder's inequality and Young's inequality, one has than we have

$$
\begin{gathered}
-\int_{0}^{t} g^{\prime}(t-\tau) \int_{\Omega} \nabla u^{n}(\tau) \cdot \nabla u_{t}^{n}(t) d x d \tau \leq \frac{1}{2}\left\|\nabla u_{t}^{n}(t)\right\|_{2}^{2} \\
+\frac{\xi_{1}^{2}\|g\|_{L^{1}}}{2} \int_{0}^{t} g(t-\tau)\left\|\nabla u^{n}(\tau)\right\|_{2}^{2} d \tau .
\end{gathered}
$$

Since $\mu(t) \geq m_{0}$ and from (3.7) we obtain

$$
\begin{gathered}
-g(0) \int_{\Omega} \nabla u^{n}(t) \cdot \nabla u_{t}^{n}(t) d x \leq \frac{1}{2}\left\|\nabla u_{t}^{n}(t)\right\|_{2}^{2}+\frac{g(0)^{2}}{2}\left\|\nabla u^{n}(t)\right\|_{2}^{2} \\
\leq \frac{1}{2}\left\|\nabla u_{t}^{n}(t)\right\|_{2}^{2}+\frac{g(0)^{2} L_{1}}{2 m_{0}} .
\end{gathered}
$$

Since $\chi(t)$ is $C^{1}$-function on $[0, \infty)$ and using (3.7) we infer that

$$
\frac{\chi^{\prime}(t)}{2}\left\|u_{t}^{n}(t)\right\|_{2}^{2} \leq \frac{A_{1}}{2}\left\|u_{t}^{n}(t)\right\|_{2}^{2} \leq \frac{A_{1}}{2} L_{1} .
$$

Moreover,

$$
\begin{aligned}
\left|\mu^{\prime}(t) \int_{\Omega} \nabla u^{n}(t) \cdot \nabla u_{t}^{n}(t) d x\right| & \leq \frac{1}{2}\left\|\nabla u_{t}^{n}(t)\right\|_{2}^{2}+\frac{M_{1}^{2}}{2}\left\|\nabla u^{n}(t)\right\|_{2}^{2} \\
& \leq \frac{1}{2}\left\|\nabla u_{t}^{n}(t)\right\|_{2}^{2}+\frac{M_{1}^{2} L_{1}}{2 m_{0}},
\end{aligned}
$$


where $M_{1}=\sup _{0 \leq t \leq T}\left\{\left|\mu^{\prime}(t)\right|\right\}$ and $A_{1}=\max _{0 \leq t \leq T}\left\{\left|\chi^{\prime}(t)\right|\right\}$. Then, by using (3.9) - (3.12), we obtain from $(3.8)$

$$
\begin{aligned}
& \frac{d}{d t}\left[\mu(t) \int_{\Omega} \nabla u^{n}(t) \cdot \nabla u_{t}^{n}(t) d x+\frac{\alpha}{2}\left\|\nabla u_{t}^{n}(t)\right\|_{2}^{2}+\frac{\chi(t)}{2}\left\|u_{t}^{n}(t)\right\|_{2}^{2}\right] \\
& \quad+\frac{1}{2}\left\|u_{t t}^{n}(t)\right\|_{2}^{2} \leq c_{2}+\frac{\xi_{1}^{2}\|g\|_{L^{1}}}{2} \int_{0}^{t} g(t-\tau)\left\|\nabla u^{n}(\tau)\right\|_{2}^{2} d \tau \\
& \quad+\frac{d}{d t}\left(\int_{0}^{t} g(t-\tau) \int_{\Omega} \nabla u^{n}(\tau) \cdot \nabla u_{t}^{n}(t) d x d \tau\right) \\
& \quad+\left(\frac{3}{2}+M_{2}\right)\left\|\nabla u_{t}^{n}(t)\right\|_{2}^{2},
\end{aligned}
$$

where $c_{2}=\left(\frac{g(0)^{2}+M_{1}^{2}+A_{1} m_{0}}{2 m_{0}}\right) L_{1}+\frac{1}{2}\left\|f_{1}\right\|_{2}^{2}$ and $M_{2}=\sup _{0 \leq t \leq T}\{|\mu(t)|\}$. Thus, integrating (3.13) over $(0, t)$, we obtain

$$
\begin{aligned}
& \frac{\alpha}{2}\left\|\nabla u_{t}^{n}(t)\right\|_{2}^{2}+\frac{1}{2} \int_{0}^{t}\left\|u_{\tau \tau}^{n}(\tau)\right\|_{2}^{2} d t+\frac{\chi(t)}{2}\left\|u_{t}^{n}(t)\right\|_{2}^{2} \\
& \quad \leq c_{3}+\mu(t)\left|\int_{\Omega} \nabla u^{n}(t) \cdot \nabla u_{t}^{n}(t) d x\right|+\mu(0)\left|\int_{\Omega} \nabla u_{0}^{n} \cdot \nabla u_{1}^{n} d x\right| \\
& \quad+\left(M_{2}+\frac{3}{2}\right) \int_{0}^{t}\left\|\nabla u_{\tau}^{n}(\tau)\right\|_{2}^{2} d \tau+\int_{0}^{t} g(t-\tau) \int_{\Omega} \nabla u^{n}(\tau) \cdot \nabla u_{t}^{n}(t) d x d \tau,
\end{aligned}
$$

where $\left(c_{3}=c_{2}+\xi_{1}^{2}\|g\|_{L^{1}}^{2} L_{1}\right) T+\frac{\alpha}{2}\left\|\nabla u_{1}^{n}\right\|_{2}^{2}+\frac{\chi(0)}{2}\left\|u_{1}^{n}\right\|_{2}^{2}$. We note that using the inequality $a b \leq \frac{1}{4 \eta} a^{2}+\eta b^{2}$, where $\eta>0$ is arbitrary, it follows that

$$
\begin{aligned}
\int_{0}^{t} g & (t-\tau) \int_{\Omega} \nabla u^{n}(\tau) \cdot \nabla u_{t}^{n}(t) d x d \tau \leq \eta\left\|\nabla u_{t}^{n}(t)\right\|_{2}^{2} \\
& +\frac{1}{4 \eta}\|g\|_{L^{1}(0, \infty)}\|g\|_{L^{\infty}(0, \infty)} \int_{0}^{t}\left\|\nabla u^{n}(\tau)\right\|_{2}^{2} d \tau \leq \eta\left\|\nabla u_{t}^{n}(t)\right\|_{2}^{2} \\
& +\frac{\|g\|_{L^{1}(0, \infty)}\|g\|_{L^{\infty}(0, \infty)}}{4 \eta m_{0}} L_{1} T
\end{aligned}
$$

and

$$
\begin{aligned}
\mu(t)\left|\int_{\Omega} \nabla u^{n}(t) \cdot \nabla u_{t}^{n}(t) d x\right| & \leq \eta\left\|\nabla u_{t}^{n}(t)\right\|_{2}^{2}+\frac{M_{2}^{2}}{4 \eta}\left\|\nabla u^{n}(t)\right\|_{2}^{2} \\
& \leq \eta\left\|\nabla u_{t}^{n}(t)\right\|_{2}^{2}+\frac{M_{2}^{2}}{4 \eta m_{0}} L_{1} .
\end{aligned}
$$

By plugging (3.15) and (3.16) into (3.14) with $0<\eta \leq \frac{\alpha}{4}$, we obtain from $\chi(t) \geq 0$ that

$$
\begin{aligned}
\left(\frac{\alpha}{2}-2 \eta\right)\left\|\nabla u_{t}^{n}(t)\right\|_{2}^{2} & +\frac{1}{2} \int_{0}^{t}\left\|u_{\tau \tau}^{n}(\tau)\right\|_{2}^{2} d t \leq c_{4} \\
& +\left(M_{2}+\frac{3}{2}\right) \int_{0}^{t}\left\|\nabla u_{\tau}^{n}(\tau)\right\|_{2}^{2} d \tau
\end{aligned}
$$

where

$$
c_{4}=c_{3}+\mu(0)\left\|\nabla u_{0}^{n}\right\|_{2}\left\|\nabla u_{1}^{n}\right\|_{2}+\frac{M_{2}^{2}}{4 \eta m_{0}} L_{1}+\frac{\|g\|_{L^{1}}\|g\|_{L^{\infty}}}{4 \eta m_{0}} L_{1} T .
$$


Taking into account (3.3) - (3.4), we obtain from Gronwall's Lemma the second priori estimate

$$
\left\|\nabla u_{t}^{n}(t)\right\|_{2}^{2}+\int_{0}^{t}\left\|u_{\tau \tau}^{n}(\tau)\right\|_{2}^{2} d \tau \leq L_{2}
$$

for all $t \in[0, T]$. Here $L_{2}$ is a positive constant independent of $n \in \mathbb{N}$ and $t \in[0, T]$.

Step 3. (The third priori estimate) Replacing $\omega$ by $-\Delta u^{n}(t)$ in (3.2), we have

$$
\begin{aligned}
\frac{d}{d t}[ & \left.-\int_{\Omega} u_{t}^{n}(t) \Delta u^{n}(t) d x+\frac{\alpha}{2}\left\|\Delta u^{n}(t)\right\|_{2}^{2}+\frac{\chi(t)}{2}\left\|\nabla u^{n}(t)\right\|_{2}^{2}\right] \\
& -\left\|\nabla u_{t}^{n}(t)\right\|_{2}^{2}+\mu(t)\left\|\Delta u^{n}(t)\right\|_{2}^{2} \\
& =\frac{\chi^{\prime}(t)}{2}\left\|\nabla u^{n}(t)\right\|_{2}^{2}+\int_{0}^{t} g(t-\tau) \int_{\Omega} \Delta u^{n}(\tau) \cdot \Delta u^{n}(t) d x d \tau \\
& +\int_{\Omega} f_{1}(x, t)\left(-\Delta u^{n}(t)\right) d x \leq \frac{A_{1}}{2}\left\|\nabla u^{n}(t)\right\|_{2}^{2}+2 \eta\left\|\Delta u^{n}(t)\right\|_{2}^{2} \\
& +\frac{\|g\|_{L^{1}}}{4 \eta} \int_{0}^{t} g(t-\tau)\left\|\Delta u^{n}(\tau)\right\|_{2}^{2} d \tau+\frac{1}{4 \eta}\left\|f_{1}\right\|_{2}^{2},
\end{aligned}
$$

where $0<\eta \leq \frac{m_{0}}{2}$ is some positive constant. From $\mu(t) \geq m_{0}>0$, we deduce by integration

$$
\begin{aligned}
& \frac{\alpha}{2}\left\|\Delta u^{n}(t)\right\|_{2}^{2}+\left(m_{0}-2 \eta\right) \int_{0}^{t}\left\|\Delta u^{n}(\tau)\right\|_{2}^{2} d \tau+\frac{\chi(t)}{2}\left\|\nabla u^{n}(t)\right\|_{2}^{2} \\
& \leq \int_{0}^{t}\left\|\nabla u_{\tau}^{n}(\tau)\right\|_{2}^{2} d t+\frac{A_{1}}{2} \int_{0}^{t}\left\|\nabla u^{n}(\tau)\right\|_{2}^{2} d \tau+\left|\int_{\Omega} u_{t}^{n}(t) \Delta u^{n}(t) d x\right| \\
& \quad+\left|\int_{\Omega} u_{t}^{n}(0) \Delta u^{n}(0) d x\right|+\frac{1}{4 \eta} \int_{0}^{t}\left\|f_{1}\right\|_{2}^{2} d t \\
& \quad+\frac{\alpha}{2}\left\|\Delta u_{0}^{n}\right\|_{2}^{2}+\frac{\chi(0)}{2}\left\|\nabla u_{0}^{n}\right\|_{2}^{2}+\frac{\|g\|_{L^{1}}^{2}}{4 \eta} \int_{0}^{t}\left\|\Delta u^{n}(\tau)\right\|_{2}^{2} d \tau \\
& \leq c_{5}+\left|\int_{\Omega} u_{t}^{n}(t) \Delta u^{n}(t) d x\right|+\frac{\|g\|_{L^{1}}^{2}}{4 \eta} \int_{0}^{t}\left\|\Delta u^{n}(\tau)\right\|_{2}^{2} d \tau,
\end{aligned}
$$

where

$$
c_{5}=\left\|u_{1}^{n}\right\|_{2}\left\|\Delta u_{0}^{n}\right\|_{2}+\frac{\alpha}{2}\left\|\Delta u_{0}^{n}\right\|_{2}^{2}+\frac{1}{4 \eta} \int_{0}^{t}\left\|f_{1}\right\|_{2}^{2} d \tau+\frac{\chi(0)}{2}\left\|\nabla u_{0}^{n}\right\|_{2}^{2}+\left(\frac{A_{1}}{m_{0}} L_{1}+L_{2}\right) T .
$$

We note that using the inequality $a b \leq \frac{1}{4} a^{2}+b^{2}$, it follows that

$$
\int_{\Omega} u_{t}^{n}(t) \Delta u^{n}(t) d x \leq \frac{1}{4}\left\|\Delta u^{n}(t)\right\|_{2}^{2}+\left\|u_{t}^{n}(t)\right\|_{2}^{2} .
$$

Plugging (3.22) into (3.21), we obtain from $\chi(t) \geq m_{1}>0$ that

$$
\begin{aligned}
\left(\frac{\alpha}{2}-\frac{1}{4}\right)\left\|\Delta u^{n}(t)\right\|_{2}^{2}+\left(m_{0}-2 \eta\right) \int_{0}^{t}\left\|\Delta u^{n}(\tau)\right\|_{2}^{2} d \tau \\
+\frac{\chi(t)}{2}\left\|\nabla u^{n}(t)\right\|_{2}^{2} \leq c_{6}+\frac{\|g\|_{L^{1}}^{2}}{4 \eta} \int_{0}^{t}\left\|\Delta u_{m}(\tau)\right\|_{2}^{2} d \tau
\end{aligned}
$$

where

$$
c_{6}=c_{5}+L_{1} \text {. }
$$


Taking into account $(3.3)-(3.4)$, we obtain from Gronwall's Lemma the third priori estimate,

$$
\left\|\Delta u^{n}(t)\right\|_{2}^{2}+\int_{0}^{t}\left\|\Delta u^{n}(\tau)\right\|_{2}^{2} d \tau \leq L_{3},
$$

for all $t \in[0, T]$ and $L_{3}$ is a positive constant independent of $n \in \mathbb{N}$ and $t \in[0, T]$.

Step 4. Let $p \geq n$ be two natural numbers, and consider $z^{n}=u^{p}-u^{n}$. Then, applying the same way as in the estimate step 1 and step 3 and observing that $\left\{u_{0}^{n}\right\}$ and $\left\{u_{1}^{n}\right\}$ are Cauchy sequence in $H_{0}^{1}(\Omega) \cap H^{2}(\Omega)$ and $H_{0}^{1}(\Omega)$, respectively, we deduce for all $t \in[0, T]$

$$
\left\|z_{t}^{n}(t)\right\|_{2}^{2}+\mu(t)\left\|\nabla z^{n}(t)\right\|_{2}^{2}+\int_{0}^{t}\left\|\nabla z_{\tau}^{n}(\tau)\right\|_{2}^{2} d \tau \rightarrow 0
$$

and

$$
\left\|\Delta z^{n}(t)\right\|_{2}^{2}+\int_{0}^{t}\left\|\Delta z^{n}(\tau)\right\|_{2}^{2} d \tau \rightarrow 0, \quad \text { as } \quad n \rightarrow \infty .
$$

Therefore, (3.7), (3.19), (3.24), (3.25) and (3.26), we see that

$$
\begin{array}{ccc}
u^{n} \rightarrow u \text { strongly in } & C\left(0, T ; H_{0}^{1}(\Omega)\right), \\
u_{t}^{n} \rightarrow u_{t} \text { strongly in } & C\left(0, T ; L^{2}(\Omega)\right) . \\
u_{t}^{n} \rightarrow u_{t} \text { strongly in } & L^{2}\left(0, T ; H_{0}^{1}(\Omega)\right), \\
u_{t t}^{n} \rightarrow u_{t t} \text { weakly in } & L^{2}\left(0, T ; L^{2}(\Omega)\right) .
\end{array}
$$

Then $(3.27)-(3.30)$ are sufficient to pass the limit in (3.2) to obtain in $L^{2}\left(0, T ; H^{-1}(\Omega)\right)$

$$
u_{t t}-\mu(t) \Delta u+\int_{0}^{t} g(t-\tau) \Delta u(\tau) d \tau-\alpha \Delta u_{t}+\chi(t) u_{t}=f_{1}(x, t) .
$$

Next, we want to show the uniqueness of (3.1). Let $u^{(1)}$ and $u^{(2)}$ be two solutions of (3.1). Then $y=u^{(1)}-u^{(2)}$ satisfies for $\omega \in H_{0}^{1}(\Omega)$

$$
\begin{aligned}
& \mu(t) \int_{\Omega} \nabla y(t) \cdot \nabla \omega d x-\int_{0}^{t} g(t-\tau) \int_{\Omega} \nabla y(\tau) \cdot \nabla \omega d x d \tau \\
& +\int_{\Omega} y_{t t}(t) \omega d x+\alpha \int_{\Omega} \nabla y_{t}(t) \cdot \nabla \omega d x+\chi(t) \int_{\Omega} y_{t}(t) \omega d x=0 \\
& y(x, 0)=0, \quad y_{t}(x, 0)=0, \quad x \in \Omega \\
& y(x, t)=0, \quad x \in \partial \Omega, t \geq 0 .
\end{aligned}
$$

Setting $w=2 y_{t}(t)$ in (3.32), then as in deriving (3.7), we see that

$$
\begin{aligned}
& \left\|y_{t}(t)\right\|_{2}^{2}+\mu(t)\|\nabla y(t)\|_{2}^{2}+(2 \alpha-1) \int_{0}^{t}\left\|\nabla y_{\tau}(\tau)\right\|_{2}^{2} d \tau \\
& \leq \int_{0}^{t}\left[1+\frac{1}{\mu(\tau)}\left(\left|\mu^{\prime}(\tau)\right|+\|g\|_{L^{1}}^{2}\right)\right]\left[\left\|y_{\tau}(\tau)\right\|_{2}^{2}+\mu(\tau)\|\nabla y(\tau)\|_{2}^{2}\right] d \tau .
\end{aligned}
$$

Thus employing Gronwall's Lemma, we conclude that

$$
\left\|y_{t}(t)\right\|_{2}=\|\nabla y(t)\|_{2}=0 \quad \text { for all } \quad t \in[0, T] .
$$

Therefore, we have the uniqueness. This finishes the proof of Lemma 3.1.

Now, let us prove the local existence of the problem (1.1). 
Theorem 3.2. Assume that $(A 1),(A 2)$ and $(A 3)$ are fulfilled. Suppose that $u_{0} \in$ $H^{2}(\Omega) \cap H_{0}^{1}(\Omega), u_{1} \in H_{0}^{1}(\Omega)$ be given. Then there exists a unique solution $u$ of $(1.1)$ satisfying $u \in C\left([0, T] ; H^{2}(\Omega) \cap H_{0}^{1}(\Omega)\right)$ and $u_{t} \in C\left([0, T] ; L^{2}(\Omega)\right) \cap L^{2}\left(0, T ; H_{0}^{1}(\Omega)\right)$, and at least one of the following statements is valid:

$$
\begin{aligned}
& \text { (i) } T=\infty \\
& \text { (ii) } e(u(t)) \equiv\left\|u_{t}(t)\right\|_{2}^{2}+\|\Delta u(t)\|_{2}^{2} \rightarrow \infty \text { as } t \rightarrow T^{-} .
\end{aligned}
$$

Proof. Define the following two-parameter space:

$$
X_{T, R_{0}}=\left\{\begin{array}{l}
v \in C\left([0, T] ; H_{0}^{1}(\Omega) \cap H^{2}(\Omega)\right), \\
v_{t} \in C\left([0, T] ; L^{2}(\Omega)\right) \cap L^{2}\left(0, T ; H_{0}^{1}(\Omega)\right): \\
e(v(t)) \leq R_{0}^{2}, t \in[0, T], \quad \text { with } \quad v(0)=u_{0}, \quad v_{t}(0)=u_{1} .
\end{array}\right\}
$$

for $T>0, R_{0}>0$. Then $X_{T, R_{0}}$ is a complete metric space with the distance

$$
d(y, z)=\sup _{0 \leq t \leq T} e(y(t)-z(t))^{\frac{1}{2}},
$$

where $y, z \in X_{T, R_{0}}$. Given $v \in X_{T, R_{0}}$, we consider the following problem

$$
\left\{\begin{array}{l}
u_{t t}-\psi\left(\|\nabla v\|_{2}^{2}\right) \Delta u-\alpha \Delta u_{t}+\int_{0}^{t} g(t-\tau) \Delta u(\tau) d \tau \\
+M\left(\|\nabla v\|_{2}^{2}\right) u_{t}=f(v), \quad \text { in } \quad \Omega \times(0, T) \\
u(x, 0)=u_{0}(x), \quad u_{t}(x, 0)=u_{1}(x), \quad x \in \Omega \\
u(x, t)=0, \quad x \in \partial \Omega, t \geq 0 .
\end{array}\right.
$$

By $(A 2)$, we see that $f(v) \in L^{2}\left(0, T ; L^{2}(\Omega)\right)$. Thus, by Lemma 3.1, we derive that problem (3.37) admits a unique solution $u \in C\left([0, T] ; H^{2}(\Omega) \cap H_{0}^{1}(\Omega)\right)$ and $u_{t} \in$ $C\left([0, T] ; L^{2}(\Omega)\right) \cap L^{2}\left(0, T ; H_{0}^{1}(\Omega)\right)$. Then, we define the nonlinear mapping $S v=u$, and we would like to show that there exist $T>0$ and $R_{0}>0$ such that $S$ is a contraction mapping from $X_{T, R_{0}}$ into itself. For this, we multiply the first equation of (3.37) by $2 u_{t}$ and integrate it over $\Omega$ to get

$$
\begin{aligned}
\frac{d}{d t}[ & \left.\left(\psi\left(\|\nabla v\|_{2}^{2}\right)-\int_{0}^{t} g(\tau) d \tau\right)\|\nabla u(t)\|_{2}^{2}+(g \circ \nabla u)(t)\right] \\
& +\frac{d}{d t}\left[\left\|u_{t}(t)\right\|_{2}^{2}\right]+2 \alpha\left\|\nabla u_{t}(t)\right\|_{2}^{2}+2 M\left(\|\nabla v\|_{2}^{2}\right)\left\|u_{t}(t)\right\|_{2}^{2} \\
& -\left(g^{\prime} \circ \nabla u\right)(t)+g(t)\|\nabla u(t)\|_{2}^{2} \\
& =\left(\frac{d}{d t} \psi\left(\|\nabla v\|_{2}^{2}\right)\right)\|\nabla u(t)\|_{2}^{2}+2 \int_{\Omega} f(v) u_{t} d x .
\end{aligned}
$$

The equality in (3.38) is obtained, because

$$
\begin{aligned}
& -2 \int_{0}^{t} \int_{\Omega} g(t-\tau) \nabla u(\tau) \cdot \nabla u_{t}(t) d x d \tau=-\left(g^{\prime} \circ \nabla u\right)(t) \\
& +g(t)\|\nabla u(t)\|_{2}^{2}+\frac{d}{d t}\left[(g \circ \nabla u)(t)-\int_{0}^{t} g(\tau)\|\nabla u(t)\|_{2}^{2} d \tau\right],
\end{aligned}
$$

where

$$
(g \circ \nabla u)(t)=\int_{0}^{t} g(t-\tau) \int_{\Omega}|\nabla u(\tau)-\nabla u(t)|^{2} d x d \tau
$$


Next, multiplying the first equation of (3.37) by $-2 \Delta u$, and integrating it over $\Omega$, we have

$$
\begin{gathered}
\frac{d}{d t}\left[\alpha\|\Delta u(t)\|_{2}^{2}-2 \int_{\Omega} u_{t} \Delta u d x+M\left(\|\nabla v\|_{2}^{2}\right)\|\nabla u(t)\|_{2}^{2}\right] \\
+2 \psi\left(\|\nabla v\|_{2}^{2}\right)\|\Delta u(t)\|_{2}^{2}-2\left\|\nabla u_{t}(t)\right\|_{2}^{2} \\
=\left(\frac{d}{d t} M\left(\|\nabla v\|_{2}^{2}\right)\right)\|\nabla u(t)\|_{2}^{2}-2 \int_{\Omega} f(v) \Delta u d x \\
\quad+2 \int_{0}^{t} g(t-\tau) \Delta u(\tau) \cdot \Delta u(t) d x d \tau .
\end{gathered}
$$

Multiplying (3.40) by $\epsilon, 0 \leq \epsilon \leq 1$, adding (3.38) together and taking into account $(A 1)$ and $(A 3)$, we obtain

$$
\frac{d}{d t} e^{*}(u(t))+2(\alpha-\epsilon)\left\|\nabla u_{t}(t)\right\|_{2}^{2}+2 \epsilon \psi\left(\|\nabla v\|_{2}^{2}\right)\|\Delta u(t)\|_{2}^{2} \leq I_{1}+I_{2}+I_{3},
$$

where

and

$$
\begin{aligned}
& e^{*}(u(t))=\left\|u_{t}(t)\right\|_{2}^{2}+\left(\psi\left(\|\nabla v\|_{2}^{2}\right)-\int_{0}^{t} g(\tau) d \tau\right)\|\nabla u(t)\|_{2}^{2} \\
&+(g \circ \nabla u)(t)+\epsilon \alpha\|\Delta u(t)\|_{2}^{2}-2 \epsilon \int_{\Omega} u_{t} \Delta u d x \\
&+\epsilon M\left(\|\nabla v\|_{2}^{2}\right)\|\nabla u(t)\|_{2}^{2} . \\
& I_{1}=2 \int_{\Omega} f(v)\left(u_{t}-\epsilon \Delta u\right) d x, \\
& I_{2}=\left(\frac{d}{d t} \psi\left(\|\nabla v\|_{2}^{2}\right)+\epsilon \frac{d}{d t} M\left(\|\nabla v\|_{2}^{2}\right)\right)\|\nabla u(t)\|_{2}^{2},
\end{aligned}
$$

$$
I_{3}=2 \epsilon \int_{0}^{t} g(t-\tau) \Delta u(\tau) \cdot \Delta u(t) d x d \tau .
$$

Estimate for $I_{1}=2 \int_{\Omega} f(v)\left(u_{t}-\epsilon \Delta u\right) d x$. From $(A 2)$ and making use of Hölder's inequality and Lemma 2.1, we have

$$
\begin{aligned}
I_{1} & =2 \int_{\Omega} f(v)\left(u_{t}-\epsilon \Delta u\right) d x \\
& \leq 2 \int_{\Omega}\left|f(v) u_{t}\right| d x+2 \epsilon \int_{\Omega}|f(v) \Delta u| d x \\
& \leq 2 K_{3} \int_{\Omega}|v|^{p-1}\left|u_{t}\right| d x+2 \epsilon K_{3} \int_{\Omega}|v|^{p-1}|\Delta u| d x \\
& \leq 2 K_{3} B_{1}^{2(p-1)}\|\Delta v\|_{2}^{p-1}\left\|u_{t}\right\|_{2}+2 \epsilon K_{3} B_{1}^{2(p-1)}\|\Delta v\|_{2}^{p-1}\|\Delta u\|_{2} \\
& \leq 2 K_{3} B_{1}^{2(p-1)} R_{0}^{p-1} e(u(t))^{\frac{1}{2}}+2 \epsilon K_{3} B_{1}^{2(p-1)} R_{0}^{p-1} e(u(t))^{\frac{1}{2}} \\
& =2 K_{3}(1+\epsilon) B_{1}^{2(p-1)} R_{0}^{p-1} e(u(t))^{\frac{1}{2}} .
\end{aligned}
$$

Estimate for $I_{2}=\left(\frac{d}{d t} \psi\left(\|\nabla v\|_{2}^{2}\right)+\epsilon \frac{d}{d t} M\left(\|\nabla v\|_{2}^{2}\right)\right)\|\nabla u(t)\|_{2}^{2}$. First of all, we observe that

$$
\begin{aligned}
\frac{d}{d t} \psi\left(\|\nabla v\|_{2}^{2}\right) & =2 \psi^{\prime}\left(\|\nabla v\|_{2}^{2}\right) \int_{\Omega} \nabla v \cdot \nabla v_{t} d x \\
& \leq 2 M_{3}\|\Delta v\|_{2}\left\|v_{t}\right\|_{2} \leq 2 M_{3} R_{0}^{2}
\end{aligned}
$$


where $M_{3}=\sup \left\{\left|\psi^{\prime}(s)\right| ; 0 \leq s \leq B_{1}^{2} R_{0}^{2}\right\}$, and

$$
\begin{aligned}
\epsilon \frac{d}{d t} M\left(\|\nabla v\|_{2}^{2}\right) & =2 \epsilon M^{\prime}\left(\|\nabla v\|_{2}^{2}\right) \int_{\Omega} \nabla v \cdot \nabla v_{t} d x \\
& \leq 2 \epsilon A_{2}\|\Delta v\|_{2}\left\|v_{t}\right\|_{2} \leq 2 \epsilon A_{2} R_{0}^{2}
\end{aligned}
$$

where $A_{2}=\max \left\{\left|M^{\prime}(s)\right| ; 0 \leq s \leq B_{1}^{2} R_{0}^{2}\right\}$. Then, from (3.44),(3.45) and using (3.35) we arrive at

$$
I_{2} \leq 2 B_{1}^{2} R_{0}^{2}\left(M_{3}+\epsilon A_{2}\right) e(u(t)) .
$$

Estimate for $I_{3}=2 \epsilon \int_{0}^{t} g(t-\tau) \Delta u(\tau) \cdot \Delta u(t) d x d \tau$. Using the inequality $a b \leq \frac{1}{4 \eta} a^{2}+\eta b^{2}$, where $\eta>0$ is arbitrary, we get

$$
\begin{aligned}
I_{3} & =2 \epsilon \int_{0}^{t} g(t-\tau) \int_{\Omega} \Delta u(\tau) \cdot \Delta u(t) d x d \tau \\
& \leq 2 \epsilon \eta\|\Delta u(t)\|_{2}^{2}+\epsilon \frac{\|g\|_{L^{1}}}{2 \eta} \int_{0}^{t} g(t-\tau)\|\Delta u(\tau)\|_{2}^{2} d \tau .
\end{aligned}
$$

Combining these inequalities with $0<\eta<\frac{\|g\|_{L^{1}}}{2}$, we get

$$
\begin{aligned}
& \frac{d}{d t} e^{*}(u(t))+2(\alpha-\epsilon)\left\|\nabla u_{t}(t)\right\|_{2}^{2}+2 \epsilon\left(\psi\left(\|\nabla v\|_{2}^{2}\right)-\eta\right)\|\Delta u(t)\|_{2}^{2} \\
& \quad \leq 2 B_{1}^{2} R_{0}^{2}\left(M_{3}+\epsilon A_{2}\right) e(u(t))+2 K_{3}(1+\epsilon) B_{1}^{2(p-1)} R_{0}^{p-1} e(u(t))^{\frac{1}{2}} \\
& \quad+\epsilon \frac{\|g\|_{L^{1}}}{2 \eta} \int_{0}^{t} g(t-\tau)\|\Delta u(\tau)\|_{2}^{2} d \tau .
\end{aligned}
$$

When we take $\epsilon=0$ in (3.48), we see that

$$
\begin{aligned}
\frac{d}{d t}[ & \left.\left(\psi\left(\|\nabla v\|_{2}^{2}\right)-\int_{0}^{t} g(\tau) d \tau\right)\|\nabla u(t)\|_{2}^{2}+(g \circ \nabla u)(t)\right] \\
& +\frac{d}{d t}\left[\left\|u_{t}(t)\right\|_{2}^{2}\right]+2 \alpha\left\|\nabla u_{t}(t)\right\|_{2}^{2} \\
& \leq 2 B_{1}^{2} R_{0}^{2} M_{3} e(u(t))+2 K_{3} B_{1}^{2(p-1)} R_{0}^{p-1} e(u(t))^{\frac{1}{2}} .
\end{aligned}
$$

By Young's inequality, we get

$$
2 \epsilon \int_{\Omega} u_{t} \Delta u d x \leq 2 \epsilon\left\|u_{t}\right\|_{2}^{2}+\frac{\epsilon}{2}\|\Delta u(t)\|_{2}^{2} .
$$

Hence

$$
\begin{gathered}
e^{*}(u(t)) \geq(1-2 \epsilon)\left\|u_{t}\right\|_{2}^{2}+\epsilon\left(\alpha-\frac{1}{2}\right)\|\Delta u(t)\|_{2}^{2}+(g \circ \nabla u)(t) \\
+\epsilon M\left(\|\nabla v\|_{2}^{2}\right)\|\nabla u(t)\|_{2}^{2}+\left(\psi\left(\|\nabla v\|_{2}^{2}\right)-\int_{0}^{t} g(\tau) d \tau\right)\|\nabla u(t)\|_{2}^{2} .
\end{gathered}
$$

Choosing $\epsilon=\frac{2}{5}$ and taking into account $(A 1)$ and $(A 3)$, we have

$$
e^{*}(u(t)) \geq \frac{1}{5} e(u(t))
$$

and

$$
\begin{aligned}
& e^{*}\left(u_{0}\right) \leq(1+2 \epsilon)\left\|u_{1}\right\|_{2}^{2}+\epsilon\left(\alpha+\frac{1}{2}\right)\left\|\Delta u_{0}\right\|_{2}^{2}+\psi\left(\left\|\nabla u_{0}\right\|_{2}^{2}\right)\left\|\nabla u_{0}\right\|_{2}^{2} \\
& +\epsilon M\left(\left\|\nabla u_{0}\right\|_{2}^{2}\right)\left\|\nabla u_{0}\right\|_{2}^{2} \leq 2\left\|u_{1}\right\|_{2}^{2}+\left(\alpha+\frac{1}{2}\right)\left\|\Delta u_{0}\right\|_{2}^{2} \\
& +\psi\left(\left\|\nabla u_{0}\right\|_{2}^{2}\right)\left\|\nabla u_{0}\right\|_{2}^{2}+M\left(\left\|\nabla u_{0}\right\|_{2}^{2}\right)\left\|\nabla u_{0}\right\|_{2}^{2}=c^{*} .
\end{aligned}
$$


Integrating (3.48) over $(0, t)$, we get

$$
\begin{aligned}
& e^{*}(u(t))+\frac{4}{5}\left(m_{0}-\eta-\frac{\|g\|_{L^{1}}^{2}}{4 \eta}\right) \int_{0}^{t}\|\Delta u(\tau)\|_{2}^{2} d \tau \\
\leq & e^{*}\left(u_{0}\right)+\int_{0}^{t}\left[C_{1} e^{*}(u(\tau))+C_{2} e^{*}(u(\tau))^{\frac{1}{2}}\right] d \tau,
\end{aligned}
$$

where $C_{1}=10 B_{1}^{2} R_{0}^{2}\left(M_{3}+\frac{2}{5} A_{2}\right)$ and $C_{2}=\frac{14 \sqrt{5}}{5} K_{1} B_{1}^{2(p-1)} R_{0}^{p-1}$. Taking $\eta=\frac{\|g\|_{L^{1}}}{2 \eta}$ in (3.53), then from $(A 1)$, we deduce

$$
\begin{aligned}
& e^{*}(u(t)) \leq e^{*}\left(u_{0}\right)+\int_{0}^{t}\left[C_{1} e^{*}(u(\tau))+C_{2} e^{*}(u(\tau))^{\frac{1}{2}}\right] d \tau \\
\leq & c^{*}+\int_{0}^{t}\left[C_{1} e^{*}(u(\tau))+C_{2} e^{*}(u(\tau))^{\frac{1}{2}}\right] d \tau .
\end{aligned}
$$

Hence, by Gronwall's inequality, we have

$$
e^{*}(u(t)) \leq\left(\sqrt{c^{*}}+\frac{C_{2}}{2} T\right)^{2} e^{C_{1} T} .
$$

Then, by (3.51), we obtain

$$
e(u(t)) \leq 5\left(\sqrt{c^{*}}+\frac{C_{2}}{2} T\right)^{2} e^{C_{1} T} .
$$

for any $t \in(0, T]$. Therefore, we see that for the parameters $T$ and $R_{0}$ satisfy

$$
5\left(\sqrt{c^{*}}+\frac{C_{2}}{2} T\right)^{2} e^{C_{1} T} \leq R_{0}^{2} .
$$

That means $S$ maps $X_{T, R_{0}}$ into itself. Moreover, by Lemma 3.1,

$$
u \in C^{0}\left([0, T] ; H^{2}(\Omega) \cap H_{0}^{1}(\Omega)\right) \cap C^{1}\left([0, T] ; L^{2}(\Omega)\right) .
$$

On the other hand, it follows from (3.49) and (3.56) that

$$
u_{t} \in L^{2}\left(0, T ; H_{0}^{1}(\Omega)\right) \text {. }
$$

Next, we shall verify that $S$ is a contraction mapping with respect to the metric $d(\cdot, \cdot)$. We take $v_{1}, v_{2} \in X_{T, R_{0}}$, and denote $u^{(1)}=S v_{1}$ and $u^{(2)}=S v_{2}$. Hereafter we suppose that $(3.57)$ is valid, thus $u^{(1)}, u^{(2)} \in X_{T, R_{0}}$. Putting $w(t)=\left(u^{(1)}-u^{(2)}\right)(t)$, then $w$ satisfies

$$
\left\{\begin{array}{l}
w_{t t}-\psi\left(\left\|\nabla v_{1}\right\|_{2}^{2}\right) \Delta w+\int_{0}^{t} g(t-\tau) \Delta w(\tau) d \tau-\alpha \Delta w_{t} \\
+M\left(\left\|\nabla v_{1}\right\|_{2}^{2}\right) w_{t}=f\left(v_{1}\right)-f\left(v_{2}\right) \\
\quad+\left[\psi\left(\left\|\nabla v_{1}\right\|_{2}^{2}\right)-\psi\left(\left\|\nabla v_{2}\right\|_{2}^{2}\right)\right] \Delta u^{(2)} \\
\quad+\left[M\left(\left\|\nabla v_{2}\right\|_{2}^{2}\right)-M\left(\left\|\nabla v_{1}\right\|_{2}^{2}\right)\right] u_{t}^{(2)} \\
w(0)=0, \quad w_{t}(0)=0 \\
w(x, t)=0, \quad x \in \partial \Omega, t \geq 0
\end{array}\right.
$$


We multiply the first equation of (3.58) by $2 w_{t}$ and integrate it over $\Omega$ to get

$$
\begin{gathered}
\frac{d}{d t}\left[\left(\psi\left(\left\|\nabla v_{1}\right\|_{2}^{2}\right)-\int_{0}^{t} g(\tau) d \tau\right)\|\nabla w(t)\|_{2}^{2}+(g \circ \nabla w)(t)\right] \\
+\frac{d}{d t}\left[\left\|w_{t}(t)\right\|_{2}^{2}\right]+2 \alpha\left\|\nabla w_{t}(t)\right\|_{2}^{2} \leq I_{4}+I_{5}+I_{6}+I_{7} .
\end{gathered}
$$

We now estimate $I_{4}-I_{7}$ (defined as below), respectively.

$$
\begin{aligned}
I_{4} & =\left(\frac{d}{d t} \psi\left(\left\|\nabla v_{1}\right\|_{2}^{2}\right)\right)\|\nabla w(t)\|_{2}^{2} \leq 2 M_{3} B_{1}^{2} R_{0}^{2} e(w(t)), \\
I_{5} & =2 \int_{\Omega}\left[f\left(v_{1}\right)-f\left(v_{2}\right)\right] w_{t} d x \\
& \leq 2 K_{3} \int_{\Omega}\left(\left|v_{1}\right|^{p-2}+\left|v_{2}\right|^{p-2}\right)\left|v_{1}-v_{2}\right| w_{t} d x \\
& \leq 2 K_{3}\left[\left\|v_{1}\right\|_{N(p-2)}^{p-2}+\left\|v_{2}\right\|_{N(p-2)}^{p-2}\right]\left\|v_{1}-v_{2}\right\|_{\frac{2 N}{N-2}}\left\|w_{t}\right\|_{2} \\
& \leq 4 K_{3} B_{1}^{2(p-1)} R_{0}^{p-2} e\left(v_{1}-v_{2}\right)^{\frac{1}{2}} e(w(t))^{\frac{1}{2}}, \\
I_{6} & =2\left[\psi\left(\left\|\nabla v_{1}\right\|_{2}^{2}\right)-\psi\left(\left\|\nabla v_{2}\right\|_{2}^{2}\right)\right] \int_{\Omega} \Delta u^{(2)} w_{t} d x \\
\leq & 2 L\left(\left\|\nabla v_{1}\right\|_{2}+\left\|\nabla v_{2}\right\|_{2}\right)\left\|\nabla v_{1}-\nabla v_{2}\right\|_{2}\left\|\Delta u^{(2)}\right\|_{2}\left\|w_{t}\right\|_{2} \\
\leq & 4 L B_{1}^{2} R_{0}^{2} e\left(v_{1}-v_{2}\right)^{\frac{1}{2}} e(w(t))^{\frac{1}{2}},
\end{aligned}
$$

where $L=L(R)$ is the Lipschitz constant of $\psi(s)$ in $\left[0, R_{0}\right]$.

Estimate for $I_{7}=2\left[M\left(\left\|\nabla v_{2}\right\|_{2}^{2}\right)-M\left(\left\|\nabla v_{1}\right\|_{2}^{2}\right)\right] \int_{\Omega} u_{t}^{(2)} w_{t} d x$. Assumption (A3) gives

$$
\begin{aligned}
& \left|M\left(\left\|\nabla v_{2}\right\|_{2}^{2}\right)-M\left(\left\|\nabla v_{1}\right\|_{2}^{2}\right)\right|=\left|\int_{\left\|\nabla v_{1}\right\|_{2}^{2}}^{\left\|\nabla v_{2}\right\|_{2}^{2}} M^{\prime}(r) d r\right| \\
& \quad \leq \int_{\left\|\nabla v_{1}\right\|_{2}^{2}}^{\left\|\nabla v_{2}\right\|_{2}^{2}}\left|M^{\prime}(r)\right| d r \leq C_{*}\left|\left\|\nabla v_{2}\right\|_{2}^{2}-\left\|\nabla v_{1}\right\|_{2}^{2}\right| \\
& \quad \leq C_{*}\left(\left\|\nabla v_{1}\right\|_{2}+\left\|\nabla v_{1}\right\|_{2}\right)\left\|\nabla v_{2}-\nabla v_{1}\right\|_{2},
\end{aligned}
$$

where $C_{*}$ is a positive constant. From (3.63) and (3.35), we have

$$
\begin{aligned}
I_{7} & =2\left[M\left(\left\|\nabla v_{2}\right\|_{2}^{2}\right)-M\left(\left\|\nabla v_{1}\right\|_{2}^{2}\right)\right] \int_{\Omega} u_{t}^{(2)} w_{t} d x \\
& \leq 2 C_{*}\left(\left\|\nabla v_{1}\right\|_{2}+\left\|\nabla v_{1}\right\|_{2}\right)\left\|\nabla\left(v_{2}-v_{1}\right)\right\|_{2}\left\|u_{t}^{(2)}\right\|_{2}\left\|w_{t}\right\|_{2} \\
& \leq 2 C_{*} B_{1}^{2} R_{0}^{2} e\left(v_{1}-v_{2}\right)^{\frac{1}{2}} e(w(t))^{\frac{1}{2}} .
\end{aligned}
$$

Inserting $(3.60)-(3.64)$ in $(3.59)$, we get

$$
\begin{aligned}
& \frac{d}{d t}\left[\left(\psi\left(\left\|\nabla v_{1}\right\|_{2}^{2}\right)-\int_{0}^{t} g(\tau) d \tau\right)\|\nabla w(t)\|_{2}^{2}+(g \circ \nabla w)(t)\right] \\
& \quad \frac{d}{d t}\left[\left\|w_{t}(t)\right\|_{2}^{2}\right]+2 \alpha\left\|\nabla w_{t}(t)\right\|_{2}^{2} \\
& \quad \leq C_{3} e(w(t))+C_{4} e\left(v_{1}-v_{2}\right)^{\frac{1}{2}} e(w(t))^{\frac{1}{2}}
\end{aligned}
$$


where $C_{3}=2 M_{3} B_{1}^{2} R_{0}^{2}$ and $C_{4}=4 K_{3} B_{1}^{2(p-1)} R_{0}^{p-2}+4 L B_{1}^{2} R_{0}^{2}+2 C_{*} B_{1}^{2} R_{0}^{2}$.

On the other hand, multiplying the first equation in (3.58) by $-2 \Delta w$, and integrating it over $\Omega$, we get

$$
\begin{gathered}
\frac{d}{d t}\left\{\alpha\|\Delta w(t)\|_{2}^{2}-2 \int_{\Omega} w_{t} \Delta w d x+M\left(\left\|\nabla v_{1}\right\|_{2}^{2}\right)\|\nabla w(t)\|_{2}^{2}\right\} \\
+2 \psi\left(\left\|\nabla v_{1}\right\|_{2}^{2}\right)\|\Delta w(t)\|_{2}^{2}-2\left\|\nabla w_{t}\right\|_{2}^{2}=I_{8}+I_{9}+I_{10}+I_{11}+I_{12} .
\end{gathered}
$$

We now estimate $I_{8}-I_{11}$ (defined as below), respectively.

Applying the similar arguments as in estimating $I_{i}, i=2,3,5,6,7$, we observe that

$$
\begin{gathered}
I_{8}=\left(\frac{d}{d t} M\left(\left\|\nabla v_{1}\right\|_{2}^{2}\right)\right)\|\nabla w(t)\|_{2}^{2} \leq 2 A_{2} R_{0}^{2} B_{1}^{2} e(w(t)) \\
I_{9}=-2 \int_{\Omega}\left[f\left(v_{1}\right)-f\left(v_{2}\right)\right] \Delta w d x \\
\leq 4 K_{3} B_{1}^{2(p-1)} R_{0}^{p-2} e\left(v_{1}-v_{2}\right)^{\frac{1}{2}} e(w(t))^{\frac{1}{2}} \\
I_{10}=2\left[\psi\left(\left\|\nabla v_{1}\right\|_{2}^{2}\right)-\psi\left(\left\|\nabla v_{2}\right\|_{2}^{2}\right)\right] \int_{\Omega} \Delta u^{(2)} \Delta w d x \\
\leq 4 L B_{1}^{2} R_{0}^{2} e\left(v_{1}-v_{2}\right)^{\frac{1}{2}} e(w(t))^{\frac{1}{2}} \\
I_{11}=2\left[M\left(\left\|\nabla v_{2}\right\|_{2}^{2}\right)-M\left(\left\|\nabla v_{1}\right\|_{2}^{2}\right)\right] \int_{\Omega} \Delta u^{(2)} \Delta w d x \\
\leq 2 C_{*} B_{1}^{2} R_{0}^{2} e\left(v_{1}-v_{2}\right)^{\frac{1}{2}} e(w(t))^{\frac{1}{2}}
\end{gathered}
$$

and

$$
\begin{aligned}
I_{12} & =2 \int_{0}^{t} g(t-\tau) \int_{\Omega} \Delta w(\tau) \cdot \Delta w(t) d x d \tau \\
& \leq 2 \eta\|\Delta w(t)\|_{2}^{2}+\frac{\|g\|_{L^{1}}}{2 \eta} \int_{0}^{t} g(t-\tau)\|\Delta w(\tau)\|_{2}^{2} d \tau,
\end{aligned}
$$

where $\eta>0$ is arbitrary. Combining these inequalities with $0<\eta \leq \frac{\|g\|_{L^{1}}}{2}$, we get

$$
\begin{aligned}
\frac{d}{d t}\{ & \left.\alpha\|\Delta w(t)\|_{2}^{2}-2 \int_{\Omega} w_{t} \Delta w d x+M\left(\left\|\nabla v_{1}\right\|_{2}^{2}\right)\|\nabla w(t)\|_{2}^{2}\right\} \\
& +2\left(\psi\left(\left\|\nabla v_{1}\right\|_{2}^{2}\right)-2 \eta\right)\|\Delta w(t)\|_{2}^{2} \leq C_{4} e\left(v_{1}-v_{2}\right)^{\frac{1}{2}} e(w(t))^{\frac{1}{2}} \\
& +\frac{\|g\|_{L^{1}}}{2 \eta} \int_{0}^{t} g(t-\tau)\|\Delta w(\tau)\|_{2}^{2} d \tau+2\left\|\nabla w_{t}\right\|_{2}^{2}+C_{5} e(w(t)),
\end{aligned}
$$

where $C_{5}=2 A_{2} B_{1}^{2} R^{2}$. Multiplying (3.72) by $\epsilon, 0<\epsilon \leq 1$, and adding (3.65) together, we obtain

$$
\begin{aligned}
\frac{d}{d t} e^{* *} & (w(t))+2(\alpha-\epsilon)\left\|\nabla w_{t}\right\|_{2}^{2}+2 \epsilon\left(\psi\left(\left\|\nabla v_{1}\right\|_{2}^{2}\right)-2 \eta\right)\|\Delta w(t)\|_{2}^{2} \\
& \leq\left(C_{3}+\epsilon C_{5}\right) e(w(t))+(1+\epsilon) C_{4} e\left(v_{1}-v_{2}\right)^{\frac{1}{2}} e(w(t))^{\frac{1}{2}} \\
& +\epsilon \frac{\|g\|_{L^{1}}}{2 \eta} \int_{0}^{t} g(t-\tau)\|\Delta w(\tau)\|_{2}^{2} d \tau
\end{aligned}
$$


where

$$
\begin{aligned}
& e^{* *}(w(t))=\left\|w_{t}(t)\right\|_{2}^{2}+\left(\psi\left(\left\|\nabla v_{1}\right\|_{2}^{2}\right)-\int_{0}^{t} g(\tau) d \tau\right)\|\nabla w(t)\|_{2}^{2} \\
& \quad+(g \circ \nabla w)(t)+\epsilon \alpha\|\Delta w(t)\|_{2}^{2}-2 \epsilon \int_{\Omega} w_{t} \Delta w d x \\
& +\epsilon M\left(\left\|\nabla v_{1}\right\|_{2}^{2}\right)\|\nabla w(t)\|_{2}^{2} .
\end{aligned}
$$

By using Young's inequality on the fifth term of right hand side of (3.74), we get

$$
\begin{gathered}
e^{* *}(w(t)) \geq(1-2 \epsilon)\left\|w_{t}(t)\right\|_{2}^{2}+\epsilon\left(\alpha-\frac{1}{2}\right)\|\Delta w(t)\|_{2}^{2} \\
\quad+\left(\psi\left(\left\|\nabla v_{1}\right\|_{2}^{2}\right)-\int_{0}^{t} g(\tau) d \tau\right)\|\nabla w(t)\|_{2}^{2} \\
\quad+(g \circ \nabla w)(t)+\epsilon M\left(\left\|\nabla v_{1}\right\|_{2}^{2}\right)\|\nabla w(t)\|_{2}^{2} .
\end{gathered}
$$

Choosing $\epsilon=\frac{2}{5}$ and by (2.1), (2.4), we have

$$
e^{* *}(w(t)) \geq \frac{1}{5} e(w(t))
$$

Then, applying the some way as in obtained (3.53) and taking $\eta=\frac{\|g\|_{L^{1}}}{2 \eta}$, we deduce

$$
\begin{aligned}
& e^{* *}(w(t)) \leq \int_{0}^{t}\left[5\left(C_{3}+\frac{2}{5} C_{5}\right) e^{* *}(w(t))\right. \\
& \left.\quad+\frac{7 \sqrt{5}}{5} C_{4} e\left(v_{1}-v_{2}\right)^{\frac{1}{2}} e^{* *}(w(t))^{\frac{1}{2}}\right] d \tau+e^{* *}(w(0)) .
\end{aligned}
$$

Thus, applying Gronwall's Lemma and noting that $e^{* *}(w(0))=0$, we have

$$
e^{* *}(w(t)) \leq \frac{49}{20} C_{4}^{2} T^{2} e^{5\left(C_{3}+\frac{2}{5} C_{5}\right) T} \sup _{0 \leq t \leq T} e\left(v_{1}-v_{2}\right) .
$$

By (3.36) and (3.76), we have

$$
d\left(u^{(1)}, u^{(2)}\right) \leq C\left(T, R_{0}\right)^{\frac{1}{2}} d\left(v_{1}, v_{2}\right),
$$

where

$$
C\left(T, R_{0}\right)^{\frac{1}{2}}=\frac{49}{4} C_{4}^{2} T^{2} e^{5\left(C_{3}+\frac{2}{5} C_{5}\right) T} .
$$

Hence, under inequality (3.57), $S$ is a contraction mapping if $C\left(T, R_{0}\right)<1$. Indeed, we choose $R_{0}$ sufficient large and $T$ sufficient small so that (3.57) and (3.79) are satisfied at the same time. By applying Banach fixed point theorem, we obtain the local existence result.

The second statement of the theorem is proved by a standard continuation argument. Indeed, let $[0, T)$ be a maximal existence interval on which the solution of (1.1) exists. Suppose that $T<\infty$ and $\lim _{t \rightarrow T^{-}}\left(\left\|u_{t}(t)\right\|_{2}^{2}+\|\Delta u(t)\|_{2}^{2}\right)<\infty$. Then, there are a sequence $\left\{t_{n}\right\}$ and a constant $K>0$ such that $t_{n} \rightarrow T^{-}$as $n \rightarrow \infty$ and $\left\|u_{t}\left(t_{n}\right)\right\|_{2}^{2}+\left\|\Delta u\left(t_{n}\right)\right\|_{2}^{2} \leq K, n=1,2, \ldots$ Since for all $n \in \mathbb{N}$, there exists a unique solution of (1.1) with initial data $\left(u\left(t_{n}\right), u_{t}\left(t_{n}\right)\right)$ on $\left[t_{n}, t_{n+\rho}\right], \rho>0$ depending on $K$ and independent of $n \in \mathbb{N}$. Thus, we can get $T<t_{n}+\rho$ for $n \in \mathbb{N}$ large enough. It contradicts to the maximality of $T$. The proof of Theorem 3.2 is now completed. 


\section{Global existence and energy decay}

In this section, we consider the global existence and energy decay of solutions for a kind of the problem (1.1):

$$
\left\{\begin{array}{l}
u_{t t}-\psi\left(\|\nabla u\|_{2}^{2}\right) \Delta u-\alpha \Delta u_{t}+\int_{0}^{t} g(t-\tau) \Delta u(\tau) d \tau \\
+M\left(\|\nabla u\|_{2}^{2}\right) u_{t}=|u|^{p-2} u, \quad x \in \Omega, t>0 \\
u(x, 0)=u_{0}(x), \quad u_{t}(x, 0)=u_{1}(x), \quad x \in \Omega, \\
u(x, t)=0, \quad x \in \partial \Omega, t>0,
\end{array}\right.
$$

where $2<p \leq \frac{2(N-1)}{N-2}, \alpha \geq 1$ and $\psi(r)=1+b r^{\gamma}, b \geq 0, \gamma \geq 1$ and $r \geq 0$.

To obtain the results of this section, we now define some functionals as follows:

$$
\begin{gathered}
I_{1}(t)=I_{1}(u(t))=\left(1-\int_{0}^{t} g(\tau) d \tau\right)\|\nabla u(t)\|_{2}^{2}+(g \circ \nabla u)(t)-\|u(t)\|_{p}^{p}, \\
I_{2}(t)=I_{2}(u(t))=I_{1}(t)+b\|\nabla u(t)\|_{2}^{2(\gamma+1)}, \\
J(t)=J(u(t))=\frac{1}{2}\left(1-\int_{0}^{t} g(\tau) d \tau\right)\|\nabla u(t)\|_{2}^{2}+\frac{1}{2}(g \circ \nabla u)(t) \\
+\frac{b}{2(\gamma+1)}\|\nabla u(t)\|_{2}^{2(\gamma+1)}-\frac{1}{p}\|u(t)\|_{p}^{p} .
\end{gathered}
$$

We define the energy of the solution $u$ of (4.1) by

$$
\begin{aligned}
E(t) & =E(u(t))=\frac{1}{2}\left\|u_{t}(t)\right\|_{2}^{2}+J(u(t))=\frac{1}{2}\left\|u_{t}(t)\right\|_{2}^{2} \\
& +\frac{1}{2}\left(1-\int_{0}^{t} g(\tau) d \tau\right)\|\nabla u(t)\|_{2}^{2}+\frac{1}{2}(g \circ \nabla u)(t) \\
& +\frac{b}{2(\gamma+1)}\|\nabla u(t)\|_{2}^{2(\gamma+1)}-\frac{1}{p}\|u(t)\|_{p}^{p} .
\end{aligned}
$$

Lemma 4.1. $E(t)$ is a non-increasing function for $t \geq 0$, that is

$$
\begin{aligned}
E^{\prime}(t) & \leq-\left[m_{1}\left\|u_{t}(t)\right\|_{2}^{2}+\alpha\left\|\nabla u_{t}(t)\right\|_{2}^{2}+\frac{K_{2}}{2}(g \circ \nabla u)(t)\right. \\
& \left.+\frac{1}{2} g(t)\|\nabla u(t)\|_{2}^{2}\right] \leq 0, \text { for all } t>0 .
\end{aligned}
$$

Proof. Multiplying the differential equation in (4.1) by $u_{t}$, integrating by parts over $\Omega$ and using $(A 3)$, we obtain

$$
\begin{aligned}
& \frac{d}{d t}\left[\frac{1}{2}\left\|u_{t}(t)\right\|_{2}^{2}+\frac{1}{2}\|\nabla u(t)\|_{2}^{2}+\frac{b}{2(\gamma+1)}\|\nabla u(t)\|_{2}^{2(\gamma+1)}-\frac{1}{p}\|u(t)\|_{p}^{p}\right] \\
& \quad=-\alpha\left\|\nabla u_{t}(t)\right\|_{2}^{2}-M\left(\|\nabla u\|_{2}^{2}\right)\left\|u_{t}(t)\right\|_{2}^{2} \\
& \quad+\int_{0}^{t} \int_{\Omega} g(t-\tau) \nabla u(\tau) \cdot \nabla u_{t}(t) d x d \tau \\
& \leq-\alpha\left\|\nabla u_{t}(t)\right\|_{2}^{2}-m_{1}\left\|u_{t}(t)\right\|_{2}^{2}+\int_{0}^{t} \int_{\Omega} g(t-\tau) \nabla u(\tau) \cdot \nabla u_{t}(t) d x d \tau .
\end{aligned}
$$

Exploiting (3.39) on the third term on the right hand side of the above inequality and using $(A 1)$, we have the result. 
Lemma 4.2. Let $u$ be the solution of (4.1). Assume the conditions of Theorem 3.2 hold. If $I_{1}(0)>0$ and

$$
\sigma=\frac{B_{1}^{p}}{l_{1}}\left(\frac{2 p}{l_{1}(p-2)} E(0)\right)^{\frac{p-2}{2}}<1,
$$

then $I_{2}(t)>0$, for all $t \geq 0$.

Proof. Since $I_{1}(0)>0$, it follows from the continuity of $u(t)$ that

$$
I_{1}(t)>0,
$$

for some interval near $t=0$. Let $t_{\max }>0$ be a maximal time (possibly $t_{\max }=T$ ), when (4.8) holds on $\left[0, t_{\max }\right)$. From (4.2) and (4.4), we have

$$
\begin{aligned}
J(t) & \geq \frac{1}{2}\left(1-\int_{0}^{t} g(\tau) d \tau\right)\|\nabla u\|_{2}^{2}+\frac{1}{2}(g \circ \nabla u)(t)-\frac{1}{p}\|u\|_{p}^{p} \\
& \geq \frac{p-2}{2 p}\left[\left(1-\int_{0}^{t} g(\tau) d \tau\right)\|\nabla u\|_{2}^{2}+(g \circ \nabla u)(t)\right]+\frac{1}{p} I_{1}(t) \\
& \geq \frac{p-2}{p}\left(1-\int_{0}^{t} g(\tau) d \tau\right)\|\nabla u\|_{2}^{2} \geq\left(\frac{p-2}{2 p}\right) l_{1}\|\nabla u\|_{2}^{2} .
\end{aligned}
$$

Using (4.9), (4.5) and $E(t)$ is non-increasing by (4.6), we get

$$
l_{1}\|\nabla u\|_{2}^{2} \leq \frac{2 p}{p-2} J(t) \leq \frac{2 p}{p-2} E(t) \leq \frac{2 p}{p-2} E(0) .
$$

Exploiting Lemma 2.1 and (4.7), we obtain from (4.10) on $\left[0, t_{\max }\right)$

$$
\begin{aligned}
\|u\|_{p}^{p} & \leq B_{1}^{p}\|\nabla u\|_{2}^{p}=B_{1}^{p}\|\nabla u\|_{2}^{p-2}\|\nabla u\|_{2}^{2} \\
& \leq \frac{B_{1}^{p}}{l_{1}}\left(\frac{2 p}{l_{1}(p-2)} E(0)\right)^{\frac{p-2}{2}} l_{1}\|\nabla u\|_{2}^{2}=\sigma l_{1}\|\nabla u\|_{2}^{2} \\
& <\left(1-\int_{0}^{t} g(\tau) d \tau\right)\|\nabla u\|_{2}^{2} .
\end{aligned}
$$

Thus on $\left[0, t_{\max }\right)$, we have

$$
I_{1}(t)=\left(1-\int_{0}^{t} g(\tau) d \tau\right)\|\nabla u(t)\|_{2}^{2}+(g \circ \nabla u)(t)-\|u(t)\|_{p}^{p}>0 .
$$

This implies that we can take $t_{\max }=T$. But, from (4.2) and (4.3), we see that

$$
I_{2}(t) \geq I_{1}(t)>0, \quad t \in[0, T] .
$$

Therefore, we have $I_{2}(t)>0, t \in[0, T]$.

Next, we want to show that $T=\infty$. Multiplying the first equation in (4.1) by $-2 \Delta u$, and integrating it over $\Omega$, we get

$$
\begin{aligned}
\frac{d}{d t}\{ & \left.\alpha\|\Delta u\|_{2}^{2}-2 \int_{\Omega} u_{t} \Delta u d x+M\left(\|\nabla u\|_{2}^{2}\right)\|\nabla u\|_{2}^{2}\right\} \\
& +\left(2 \psi\left(\|\nabla u\|_{2}^{2}\right)-2 \eta\right)\|\Delta u\|_{2}^{2} \leq 2\left\|\nabla u_{t}\right\|_{2}^{2}-2 \int_{\Omega}|u|^{p-2} u \Delta u d x \\
& +\frac{\|g\|_{L^{1}}}{2 \eta} \int_{0}^{t} g(t-\tau)\|\Delta u(\tau)\|_{2}^{2} d \tau+\left(\frac{d}{d t} M\left(\|\nabla u\|_{2}^{2}\right)\right)\|\nabla u\|_{2}^{2},
\end{aligned}
$$


where $0<\eta \leq \frac{\|g\|_{L^{1}}}{2}$. On the other hand, multiplying the first equation in (4.1) by $2 u_{t}$, and integrating it over $\Omega$, we get

$$
\begin{gathered}
\frac{d}{d t}(2 E(t))+2 \alpha\left\|\nabla u_{t}\right\|_{2}^{2}=\left(g^{\prime} \circ \nabla u\right)(t)-g(t)\|\nabla u(t)\|_{2}^{2} \\
-2 M\left(\|\nabla u\|_{2}^{2}\right)\left\|u_{t}\right\|_{2}^{2} .
\end{gathered}
$$

Multiplying (4.13) by $\epsilon, 0<\epsilon \leq 1$, and adding (4.14) together, we obtain

$$
\begin{aligned}
\frac{d}{d t} E^{*}(t) & +2(\alpha-\epsilon)\left\|\nabla u_{t}\right\|_{2}^{2}+2 \epsilon\left(\psi\left(\|\nabla u\|_{2}^{2}\right)-2 \eta\right)\|\Delta u\|_{2}^{2} \\
& \leq-2 \epsilon \int_{\Omega}|u|^{\alpha-2} u \Delta u d x+\epsilon\left(\frac{d}{d t} M\left(\|\nabla u\|_{2}^{2}\right)\right)\|\nabla u\|_{2}^{2} \\
& +2 \epsilon \frac{\|g\|_{L^{1}}}{2 \eta} \int_{0}^{t} g(t-\tau)\|\Delta u(\tau)\|_{2}^{2} d \tau,
\end{aligned}
$$

where

$$
E^{*}(t)=2 E(t)-2 \epsilon \int_{\Omega} u_{t} \Delta u d x+\epsilon \alpha\|\Delta u\|_{2}^{2}+\epsilon M\left(\|\nabla u\|_{2}^{2}\right)\|\nabla u\|_{2}^{2} .
$$

By young's inequality, we get

$$
\left|2 \epsilon \int_{\Omega} u_{t} \Delta u d x\right| \leq 2 \epsilon\left\|u_{t}\right\|_{2}^{2}+\frac{\epsilon}{2}\|\Delta u\|_{2}^{2}
$$

Hence, choosing $\epsilon=\frac{2}{5}$ and by (4.11), we see that

$$
E^{*}(t) \geq \frac{1}{5}\left(\left\|u_{t}\right\|_{2}^{2}+\|\Delta u\|_{2}^{2}\right) .
$$

Let us estimate $I_{13}=\left(\frac{d}{d t} M\left(\|\nabla u\|_{2}^{2}\right)\right)\|\nabla u\|_{2}^{2}$. Since $M \in C^{1}([0, \infty)$, using $(4.10)$ and (4.18) we infer that

$$
\begin{aligned}
& I_{13}=\left(\frac{d}{d t} M\left(\|\nabla u\|_{2}^{2}\right)\right)\|\nabla u\|_{2}^{2} \\
& \quad=2 M^{\prime}\left(\|\nabla u\|_{2}^{2}\right)\left(\int_{\Omega} \nabla u \cdot \nabla u_{t} d x\right)\|\nabla u\|_{2}^{2} \\
& \leq 2 A_{3}\|\Delta u\|_{2}\left\|u_{t}\right\|_{2}\|\nabla u\|_{2}^{2} \leq 10 A_{3}\left(\frac{2 p}{l_{1}(p-2)}\right) E(0) E^{*}(t)=c_{7} E^{*}(t),
\end{aligned}
$$

where $c_{7}=10 A_{3}\left(\frac{2 p}{l_{1}(p-2)}\right) E(0)$ and $A_{3}=\max \left\{M^{\prime}(r), 0 \leq r \leq\left(\frac{2 p}{l_{1}(p-2)}\right) E(0)\right\}$. Moreover, we note that

$$
\begin{aligned}
& \left.\left.2\left|\int_{\Omega}\right| u\right|^{p-2} u \Delta u d x\left|\leq 2(p-1) \int_{\Omega}\right| u\right|^{p-2}|\nabla u|^{2} d x \\
& \leq 2(p-1)\|u\|_{(p-2) \theta_{1}}^{p-2}\|\nabla u\|_{2 \theta_{2}}^{2},
\end{aligned}
$$

where $\frac{1}{\theta_{1}}+\frac{1}{\theta_{2}}=1$, so that, we put $\theta_{1}=1$ and $\theta_{2}=\infty$, if $N=1 ; \theta_{1}=1+\epsilon_{1}$ (for arbitrary small $\epsilon_{1}>0$ ), if $N=2$; and $\theta_{2}=\frac{N}{N-2}$, if $N \geq 3$. Then, by Lemma 2.1, (4.10) and (4.18), we have

$$
\left.2\left|\int_{\Omega}\right| u\right|^{p-2} u \Delta u d x \mid \leq 2 B_{1}^{p}(p-1)\|\nabla u\|_{2}^{p-2}\|\Delta u\|_{2}^{2} \leq c_{8} E^{*}(t),
$$


where $c_{8}=10 B_{1}^{p}(p-1)\left(\frac{2 p}{l_{1}(p-2)} E(0)\right)^{\frac{p-2}{2}}$. Inserting (4.19) and (4.21) into (4.15), and then integrating it over $(0, t)$, we obtain

$$
\begin{aligned}
E^{*}(t) & +\frac{4}{5}\left(m_{0}-\eta-\frac{\|g\|_{L^{1}}^{2}}{4 \eta}\right) \int_{0}^{t}\|\Delta u(\tau)\|_{2}^{2} d \tau \\
& \leq E^{*}(0)+\int_{0}^{t} c_{9} E^{*}(\tau) d \tau
\end{aligned}
$$

where $c_{9}=c_{7}+c_{8}$. Taking $\eta=\frac{\|g\|_{L^{1}}}{2}$ in (4.22), and by Gronwall's Lemma, we deduce

$$
E^{*}(t) \leq E^{*}(0) e^{c_{9} t}
$$

for any $t \geq 0$. Therefore by Theorem 3.2, we have $T=\infty$.

Lemma 4.3. If $u$ satisfies the assumptions of Lemma 4.2, then there exists $B>0$ such that

$$
\|u\|_{p}^{p} \leq B E(t) .
$$

Proof. Using Lemma 2.1 and (4.10), we have

$$
\begin{aligned}
\|u\|_{p}^{p} & \leq B_{1}^{p}\|\nabla u\|_{2}^{p}=B_{1}^{p}\|\nabla u\|_{2}^{p-2}\|\nabla u\|_{2}^{2} \\
& \leq \frac{B_{1}^{p}}{l_{1}}\left(\frac{2 p}{l_{1}(p-2)} E(0)\right)^{\frac{p-2}{2}} l_{1}\|\nabla u\|_{2}^{2}=\sigma l_{1}\|\nabla u\|_{2}^{2} \\
& \leq \sigma\left(\frac{2 p}{p-2}\right) E(t) .
\end{aligned}
$$

Let $B=\sigma\left(\frac{2 p}{p-2}\right)$, then we have (4.24).

Theorem 4.4. (Global existence and Energy decay) Suppose that (A1) and (A3) hold. Assume $I_{1}\left(u_{0}\right)>0$ and (4.7) holds, then the problem (4.1) admits a global solution $u$ if $u_{0} \in H^{2}(\Omega) \cap H_{0}^{1}(\Omega)$ and $u_{1} \in H_{0}^{1}(\Omega)$. Moreover, we have the following decay estimates

$$
E(t) \leq c e^{-\kappa \epsilon t}, \quad \forall t \geq 0 \quad \text { and } \quad \epsilon \in\left(0, \epsilon_{1}\right],
$$

where $c, \kappa$ and $\epsilon_{1}$ are positive constants.

Proof. Defining the perturbed energy by

$$
E_{\epsilon}(t)=E(t)+\epsilon \varphi(t)
$$

where

$$
\varphi(t)=\int_{\Omega} u(t) u_{t}(t) d x,
$$

we can show that for $\epsilon$ small enough, there exist two positive constants $\beta_{1}$ and $\beta_{2}$ such that

$$
\beta_{1} E(t) \leq E_{\epsilon}(t) \leq \beta_{2} E(t)
$$

In fact

$$
\begin{aligned}
E_{\epsilon}(t) & \leq E(t)+\frac{\epsilon}{2}\left\|u_{t}\right\|_{2}^{2}+\frac{\epsilon}{2}\|u\|_{2}^{2} \leq(1+\epsilon) E(t)+\frac{\epsilon}{2} B_{1}^{2}\|\nabla u\|_{2}^{2} \\
& \leq(1+\epsilon) E(t)+\frac{\epsilon}{2} B_{1}^{2}\left(\frac{2 p}{l_{1}(p-2)}\right) E(t) \leq \beta_{2} E(t)
\end{aligned}
$$


and

$$
E_{\epsilon}(t) \geq E(t)-\frac{\epsilon}{4 \delta}\left\|u_{t}\right\|_{2}^{2}-\epsilon \delta\|u\|_{2}^{2} \geq E(t)-\frac{\epsilon}{4 \delta}\left\|u_{t}\right\|_{2}^{2}-\epsilon \delta B_{1}^{2}\|\nabla u\|_{2}^{2} .
$$

By choosing $\delta$ small enough, we have

$$
E_{\epsilon}(t) \geq E(t)-\frac{\epsilon}{4 \delta}\left\|u_{t}\right\|_{2}^{2} \geq J(u(t))+\left(\frac{1}{2}-\frac{\epsilon}{4 \delta}\right)\left\|u_{t}\right\|_{2}^{2} .
$$

Once $\delta$ is chosen, we take $\epsilon$ so small that

$$
E_{\epsilon}(t) \geq J(u(t))+\frac{\beta_{1}}{2}\left\|u_{t}\right\|_{2}^{2} \geq \beta_{1} E(t)
$$

where $\frac{\beta_{1}}{2} \leq \frac{1}{2}-\frac{\epsilon}{4 \delta}$. Now taking the derivative of $\varphi(t)$ defined in (4.26) and substituting

$$
\begin{aligned}
u_{t t} & =\psi\left(\|\nabla u\|_{2}^{2}\right) \nabla u+\alpha \Delta u_{t}-\int_{0}^{t} g(t-\tau) \Delta u(\tau) d \tau \\
& -M\left(\|\nabla u\|_{2}^{2}\right) u_{t}+|u|^{p-2} u
\end{aligned}
$$

in the obtained expression, it results that

$$
\begin{aligned}
\varphi^{\prime}(t) & =\left\|u_{t}\right\|_{2}^{2}-\|\nabla u\|_{2}^{2}-b\|\nabla u\|_{2}^{2(\gamma+1)} \\
& +\int_{0}^{t} g(t-\tau) \int_{\Omega} \nabla u(\tau) \cdot \nabla u(t) d x d \tau-\alpha\left(\nabla u_{t}, \nabla u\right) \\
& -M\left(\|\nabla u\|_{2}^{2}\right)\left(u_{t}, u\right)+\|u\|_{p}^{p} .
\end{aligned}
$$

Adding and subtracting $2 E(t)$, and taking (4.5) into account, from (4.33) we infer

$$
\begin{aligned}
\varphi^{\prime}(t) & =-2 E(t)+2\left\|u_{t}\right\|_{2}^{2}-\left(\int_{0}^{t} g(\tau) d \tau\right)\|\nabla u(t)\|_{2}^{2} \\
& +(g \circ \nabla u)(t)-b\left(1-\frac{1}{\gamma+1}\right)\|\nabla u\|_{2}^{2(\gamma+1)} \\
& +\left(1-\frac{2}{p}\right)\|u\|_{p}^{p}-\alpha\left(\nabla u_{t}, \nabla u\right)-M\left(\|\nabla u\|_{2}^{2}\right)\left(u_{t}, u\right) \\
& +\int_{0}^{t} g(t-\tau) \int_{\Omega} \nabla u(\tau) \cdot \nabla u(t) d x d \tau .
\end{aligned}
$$

Estimate for $J_{1}=\alpha\left(\nabla u_{t}, \nabla u\right)$. Considering Cauchy-Schwartz inequality, we have

$$
\left|J_{1}\right| \leq \frac{\alpha^{2}}{2}\left\|\nabla u_{t}(t)\right\|_{2}^{2}+\frac{1}{2}\|\nabla u(t)\|_{2}^{2} .
$$

Let us estimate $J_{2}=M\left(\|\nabla u\|_{2}^{2}\right)\left(u_{t}, u\right)$. Noting that $\|\nabla u(t)\|_{2}^{2} \leq \frac{2 p}{l_{1}(p-2)} E(0)=\beta_{3}$ for all $t \geq 0$, we have that

$$
M\left(\|\nabla u\|_{2}^{2}\right) \leq \xi, \quad \forall t \geq 0
$$

where $\xi=\max \left\{M(r) ; r \in\left[0, \beta_{3}\right]\right\}$. From (4.36) we conclude that

$$
\left|J_{2}\right| \leq \frac{\xi^{2}}{2}\left\|u_{t}(t)\right\|_{2}^{2}+\frac{1}{2}\|u(t)\|_{2}^{2} \leq \frac{\xi^{2}}{2}\left\|u_{t}(t)\right\|_{2}^{2}+\frac{1}{2} B_{1}^{2}\|\nabla u(t)\|_{2}^{2} .
$$


Estimate $J_{3}=\int_{0}^{t} g(t-\tau) \int_{\Omega} \nabla u(\tau) \cdot \nabla u(t) d x d \tau$. From assumption $(A 1)$ and making use of the Cauchy-Schwarz inequality, we have

$$
\begin{aligned}
J_{3} & =\int_{0}^{t} g(t-\tau) \int_{\Omega} \nabla u(\tau) \cdot \nabla u(t) d x d \tau \\
& =\int_{0}^{t} g(t-\tau) \int_{\Omega}[\nabla u(\tau)-\nabla u(t)+\nabla u(t)] \cdot \nabla u(t) d x d \tau \\
& \leq \int_{0}^{t} g(t-\tau)|\nabla u(t)-\nabla u(\tau) \| \nabla u(t)| d x d \tau \\
& +\left(\int_{0}^{t} g(\tau) d \tau\right)\|\nabla u(t)\|_{2}^{2} \\
& \leq\|\nabla u(t)\|_{2}^{2} \int_{0}^{t} g(t-\tau)\|\nabla u(t)-\nabla u(\tau)\|_{2}^{2} d \tau \\
& +\left(\int_{0}^{t} g(\tau) d \tau\right)\|\nabla u(t)\|_{2}^{2} \\
& \leq \frac{1}{2}\|\nabla u(t)\|_{2}^{2}+\frac{1}{2}\|g\|_{L^{1}(0, \infty)}(g \circ \nabla u)(t)+\left(\int_{0}^{t} g(\tau) d \tau\right)\|\nabla u(t)\|_{2}^{2} \\
& \leq \frac{1}{2}\|\nabla u(t)\|_{2}^{2}+\frac{1}{2}(g \circ \nabla u)(t)+\left(\int_{0}^{t} g(\tau) d \tau\right)\|\nabla u(t)\|_{2}^{2} .
\end{aligned}
$$

Utilizing Lemma 4.3 and inserting (4.35), (4.38) and (4.37) in (4.34), we have

$$
\begin{aligned}
\varphi^{\prime}(t) & \leq\left(\frac{\xi^{2}}{2}+2\right)\left\|u_{t}\right\|_{2}^{2}+\left(1+\frac{B_{1}^{2}}{2}\right)\|\nabla u\|_{2}^{2} \\
& +\left[\left(1-\frac{2}{p}\right) B-2\right] E(t)-b\left(1-\frac{1}{\gamma+1}\right)\|\nabla u\|_{2}^{2(\gamma+1)} \\
& +\frac{\alpha^{2}}{2}\left\|\nabla u_{t}(t)\right\|_{2}^{2}+\frac{3}{2}(g \circ \nabla u)(t) .
\end{aligned}
$$

Then, from (4.6), (4.25), (4.26) and (4.39) we arrive at

$$
\begin{aligned}
E_{\epsilon}^{\prime}(t) & =E^{\prime}(t)+\epsilon \varphi^{\prime}(t) \leq-\left(m_{1}-\lambda_{1} \epsilon\right)\left\|u_{t}\right\|_{2}^{2}+\lambda_{2} \epsilon\|\nabla u\|_{2}^{2} \\
& -\left(\frac{K_{2}}{2}-\frac{3}{2} \epsilon\right)(g \circ \nabla u)(t)-\left(\alpha-\frac{\alpha^{2}}{2} \epsilon\right)\left\|\nabla u_{t}(t)\right\|_{2}^{2} \\
& -\epsilon\left(-\lambda_{3}\right) E(t)-b \epsilon\left(1-\frac{1}{\gamma+1}\right)\|\nabla u\|_{2}^{2(\gamma+1)}-\frac{1}{2} g(t)\|\nabla u(t)\|_{2}^{2},
\end{aligned}
$$

where

and

$$
\lambda_{1}=\frac{\xi^{2}}{2}+2>0, \lambda_{2}=\frac{B_{1}^{2}}{2}+1>0
$$

$$
\lambda_{3}=\left(1-\frac{2}{p}\right) B-2=\left(1-\frac{2}{p}\right)\left(\frac{2 p}{p-2}\right) \sigma-2=2 \sigma-2<0 .
$$

On the other hand, since

$$
\int_{0}^{t} g^{\prime}(\tau) d \tau=g(t)-g(0)
$$

then

$$
-g(t)\|\nabla u(t)\|_{2}^{2}=-g(0)\|\nabla u(t)\|_{2}^{2}-\left(\int_{0}^{t} g^{\prime}(\tau) d \tau\right)\|\nabla u(t)\|_{2}^{2} .
$$


From $(A 1)$ the last inequality yields

$$
-\frac{1}{2} g(t)\|\nabla u(t)\|_{2}^{2} \leq-\frac{1}{2} g(0)\|\nabla u(t)\|_{2}^{2}+\frac{K_{1}}{2}\|g\|_{L^{1}(0, \infty)}\|\nabla u(t)\|_{2}^{2} .
$$

Combining (4.40) and (4.41) we conclude that

$$
\begin{aligned}
& E_{\epsilon}^{\prime}(t) \leq-\left(m_{1}-\lambda_{1} \epsilon\right)\left\|u_{t}\right\|_{2}^{2}-\left(\frac{K_{2}}{2}-\frac{3}{2} \epsilon\right)(g \circ \nabla u)(t) \\
& -\left(\alpha-\frac{\alpha^{2}}{2} \epsilon\right)\left\|\nabla u_{t}(t)\right\|_{2}^{2}-b \epsilon\left(1-\frac{1}{\gamma+1}\right)\|\nabla u\|_{2}^{2(\gamma+1)}-\epsilon\left(-\lambda_{3}\right) E(t) \\
& \quad-\frac{1}{2}\left[g(0)-K_{1}\|g\|_{L^{1}(0, \infty)}-2 \lambda_{2} \epsilon\right]\|\nabla u(t)\|_{2}^{2} .
\end{aligned}
$$

From (2.1) we have $l_{2}=g(0)-K_{1}\|g\|_{L^{1}(0, \infty)}>0$. Defining

$$
\epsilon_{1}=\min \left\{\frac{m_{1}}{\lambda_{1}}, \frac{K_{2}}{3}, \frac{2}{\alpha}, \frac{l_{2}}{2 \lambda_{2}}\right\},
$$

we conclude by taking $\epsilon \in\left(0, \epsilon_{1}\right]$ in $(4.42)$ that

$$
E_{\epsilon}^{\prime}(t) \leq-\epsilon\left(-\lambda_{3}\right) E(t) .
$$

Thus, we see that $\forall t \geq 0$ and $\epsilon \in\left(0, \epsilon_{1}\right]$

$$
E_{\epsilon}^{\prime}(t) \leq-\epsilon\left(-\lambda_{3}\right) E(t) \leq-\frac{-\lambda_{3}}{\beta_{2}} \epsilon E_{\epsilon}(t) .
$$

By the Gronwall inequality, we see that

$$
E_{\epsilon}(t) \leq E_{\epsilon}(0) e^{-\kappa \epsilon t}, \quad \forall t \geq 0 \text { and } \epsilon \in\left(0, \epsilon_{1}\right],
$$

where $\kappa=\frac{-\lambda_{3}}{\beta_{2}}$. Combining with (4.27), we obtain

$$
\beta_{1} E(t) \leq E_{\epsilon}(t) \leq E_{\epsilon}(0) e^{-\kappa \epsilon t}, \quad \forall t \geq 0 \text { and } \epsilon \in\left(0, \epsilon_{1}\right],
$$

and

$$
E(t) \leq c e^{-\kappa \epsilon t}, \quad \forall t \geq 0 \text { and } \epsilon \in\left(0, \epsilon_{1}\right],
$$

where $c=\frac{E_{\epsilon}(0)}{\beta_{1}}$. Thus, the proof of the theorem is completed.

\section{References}

[1] Cavalcanti, M.M., V.N. Domingos Cavalcanti, V.N., Soriano, J.A., Exponential decay of the viscoelastic Euler-Bernoulli equation with a nonlocal dissipation in general domains, Differential Integral Equations, 17(2004), 495-510.

[2] Kirchhoff, G., Vorlesungen über Mechanik, Teubner, Leipzig, 1983.

[3] Lange, H., Perla Menzala, G., Rates of decay of a nonlocal beam equation, Differential Integral Equations, 10(1997), 1075-1092.

[4] Mahdi, F.Z., Ferhat, M., Hakem, A., Blow up and asymptotic behavior for a system of viscoelastic wave equations of Kirchhoff type with a delay term, Advances in the Theory of Nonlinear Analysis and its Applications, 2(2018), no. 3, 146-167.

[5] Matsutama, T., Ikehata, R., On global solutions and energy decay for the wave equations of Kirchhoff type with nonlinear damping, J. Math. Anal. Appl., 204(1996), 729-753.

[6] Wu, S.T., Exponential energy decay of solutions for an integro-differential equation with strong damping, J. Math. Anal. Appl., 364(2010), no. 2, 609-617. 
[7] Wu, S.T., Tsai, L.Y., On global existence and blow-up of solutions for an integrodifferential equation with strong damping, Taiwanese J. Math, 10(2006), no. 4, 979-1014.

Mohamed Mellah

Laboratory ACEDP, Djillali Liabes University, 22000 Sidi Bel Abbes, Algeria

e-mail: mmellah77@yahoo.fr

Ali Hakem

Laboratory ACEDP, Djillali Liabes University, 22000 Sidi Bel Abbes, Algeria

e-mail: hakemali@yahoo.com 